\title{
A CONVERGENT FINITE DIFFERENCE METHOD FOR A NONLINEAR VARIATIONAL WAVE EQUATION
}

\author{
H. HOLDEN, K. H. KARLSEN, AND N. H. RISEBRO
}

\begin{abstract}
We establish rigorously convergence of a semi-discrete upwind scheme for the nonlinear variational wave equation $u_{t t}-c(u)\left(c(u) u_{x}\right)_{x}=0$ with $\left.u\right|_{t=0}=u_{0}$ and $\left.u_{t}\right|_{t=0}=v_{0}$. Introducing Riemann invariants $R=$ $u_{t}+c u_{x}$ and $S=u_{t}-c u_{x}$, the variational wave equation is equivalent to $R_{t}-c R_{x}=\tilde{c}\left(R^{2}-S^{2}\right)$ and $S_{t}+c S_{x}=-\tilde{c}\left(R^{2}-S^{2}\right)$ with $\tilde{c}=c^{\prime} /(4 c)$. An upwind scheme is defined for this system. We assume that the the speed $c$ is positive, increasing and both $c$ and its derivative are bounded away from zero and that $\left.R\right|_{t=0},\left.S\right|_{t=0} \in L^{1}(\mathbb{R}) \cap L^{3}(\mathbb{R})$ are nonpositive. The numerical scheme is illustrated on several examples.
\end{abstract}

\section{INTRODUCTION}

In this paper we consider the nonlinear variational wave equation

$$
\begin{gathered}
\frac{\partial^{2} u}{\partial t^{2}}-c(u) \frac{\partial}{\partial x}\left(c(u) \frac{\partial u}{\partial x}\right)=0, \\
u(x, 0)=u_{0}(x), \quad \frac{\partial u}{\partial t}(x, 0)=v_{0}(x),
\end{gathered}
$$

in the strip $(x, t) \in \Pi_{T}=\mathbb{R} \times[0, T]$.

The equation, which can be derived as the Euler-Lagrange equation for the variational principle $\delta \iint\left(\psi_{t}^{2}-c^{2}(\psi) \psi_{x}^{2}\right) d x d t=0$, can be used to model liquid crystals, see [8, 6, 3]. Consider namely a nematic liquid crystal in the regime where inertial effects dominate. In that case the liquid crystal can be described by the director field $n=n(x, y, z, t) \in \mathbb{R}^{3}$ with $\|n\|=1$ that describes the direction of the elongated molecules that constitute the liquid crystal. Its potential energy density is described by the Oseen-Franck functional

$$
W(n, \nabla n)=\alpha|n \times(\nabla \times n)|^{2}+\beta(\nabla \cdot n)^{2}+\gamma(n \cdot \nabla n)^{2},
$$

where the constants $\alpha, \beta$, and $\gamma$ describe the liquid crystal. The dynamics of the director field is given by a least action principle

$$
\frac{\delta}{\delta u} \int\left(n_{t} \cdot n_{t}-W(n, \nabla n)\right) d x d y d z d t=0 .
$$

Consider the class of planar deformations given by

$$
n=\cos (u(x, t)) \mathbf{i}+\sin (u(x, t)) \mathbf{j}
$$

Date: August 22, 2021.

2000 Mathematics Subject Classification. Primary: 35D05, 65M12; Secondary: 65M06.

Key words and phrases. Variational wave equation, convergence of finite difference schemes, liquid crystals.

This research was supported in part by the Research Council of Norway. KHK has been supported in part by an Outstanding Young Investigators Award from the Research Council of Norway. 
where $\mathbf{i}$ and $\mathbf{j}$ are unit vectors in the $x$ and $y$ direction, respectively. In that case the least action principle (1.2) reduces to (1.1) with

$$
c^{2}(u)=\alpha \cos ^{2} u+\beta \sin ^{2} u .
$$

We here analyze (1.1) with more restrictive assumptions on $c$, as is done in the mathematical literature, namely that $c$ is positive, strictly increasing and bounded away from zero. We note that (1.1) is closely related to the Hunter-Saxton equation, which is obtained by a further asymptotic expansion of (1.1), see 6 .

While short-term existence of regular solutions follows by the Kato method, it is clear that the solution in general develops singularities in finite time, even from smooth initial data, see [4, 3].

In a series of papers, Zhang and Zheng [11, 12, 13, 14, 15, have analyzed 1.1) carefully, using the method of Young measures. From their many results we quote the recent one [14, Thm. 1.1] where they show existence of a global weak solution for initial data $u_{0} \in H^{1}(\mathbb{R})$ and $v_{0} \in L^{2}(\mathbb{R})$. The function $c$ is assumed to be smooth, bounded, positive with derivative that is non-negative and strictly positive on the initial data $u_{0}$. Their results, and also the relationship to the Hunter-Saxton equation are surveyed in [15. The uniqueness question is open.

Another approach to the study of (1.1) was recently taken by Bressan and Zheng 1. Instead of following the approach based on Young measures, they rewrite the equation in new variables where singularities disappear. They show that for $u_{0}$ absolutely continuous with $u_{0, x}, v_{0} \in L^{2}(\mathbb{R})$ the Cauchy-problem 1.1 allows a global weak solution with the following properties: The solution $u$ is locally Hölder continuous with exponent $\frac{1}{2}$, and the map $t \mapsto u(t, \cdot)$ continuously differentiable with values in $L_{\text {loc }}^{p}(\mathbb{R})$ for $1 \leq p<2$. Further properties are obtained, in particular, it is shown that the associated energy, treated as a measure, is conserved in time.

Up to now, little has been known about the behavior of numerical schemes for the equation (1.1). Except for some numerical computations in [3, there are, to the best of our knowledge, no rigorous results regarding any numerical method for 1.1, and the main purpose of this paper is to remedy this situation. Here we introduce a semidiscrete upwind scheme for the initial-value problem (1.1), i.e., a finite difference approximation of the spatial variation, keeping the continuous temporal variation. For this scheme we show convergence to a weak solution of $(1.1)$, and thus this proof offers a different existence proof compared with the others. In addition it provides a constructive approach to the initial-value problem in the sense that the difference scheme supplies a numerical tool to compute the solution, see Section 4 . Indeed, we show how the difference scheme performs, both on examples where the scheme is proved to converge and otherwise. A similar analysis has been applied to the Hunter-Saxton equation, see [5].

We now turn to a more detailed and technical discussion. Weak solutions are defined as follows.

Definition 1.1. Let $\Pi_{T}$ denote the set $\mathbb{R} \times[0, T), T>0$. By a weak solution $u$ of 1.1. we mean a function $u \in L^{\infty}\left([0, T] ; W^{1, p}(\mathbb{R})\right) \cap C\left(\Pi_{T}\right), u_{t} \in L^{\infty}\left([0, T] ; L^{p}(\mathbb{R})\right)$, for all $p \in[1,3+q]$, where $q$ is some fixed positive constant $q>0$, such that

$$
\iint_{\Pi_{T}}\left(\partial_{t} \varphi \partial_{t} u-c^{2}(u) \partial_{x} \varphi \partial_{x} u-c^{\prime}(u) c(u) \varphi\left(\partial_{x} u\right)^{2}\right) d x d t=0
$$

for all test functions $\varphi \in C_{0}^{\infty}\left(\Pi_{T}\right)$. The initial values are taken in the sense that $u(\cdot, t) \rightarrow u_{0}$ in $C\left([0, T] ; L^{2}(\mathbb{R})\right)$ as $t \rightarrow 0+$, and $\partial_{t} u(\cdot, t) \rightarrow v_{0}$ as a distribution in $\Pi_{T}$ when $t \rightarrow 0+$. 
A common approach to 1.1 is first to re-write the equation in terms of Riemann invariants. To that end we define

$$
R=\frac{\partial u}{\partial t}+c(u) \frac{\partial u}{\partial x}, \quad S=\frac{\partial u}{\partial t}-c(u) \frac{\partial u}{\partial x},
$$

and the auxiliary function

$$
\tilde{c}(u)=\frac{c^{\prime}(u)}{4 c(u)} .
$$

Then the wave equation 1.1 is formally equivalent to the $3 \times 3$ system

$$
\begin{aligned}
R_{t}-c(u) R_{x} & =\tilde{c}(u)\left(R^{2}-S^{2}\right), \\
S_{t}+c(u) S_{x} & =-\tilde{c}(u)\left(R^{2}-S^{2}\right), \\
u_{x} & =\frac{1}{2 c(u)}(R-S), \\
R_{0}=\left.R\right|_{t=0} & =v_{0}+c\left(u_{0}\right) u_{0}^{\prime}, \quad S_{0}=\left.S\right|_{t=0}=v_{0}-c\left(u_{0}\right) u_{0}^{\prime} .
\end{aligned}
$$

Clearly, we also have

$$
u_{t}=\frac{1}{2}(R+S) .
$$

In order to make this well defined, we use the boundary condition

$$
\lim _{x \rightarrow-\infty} u(x, t)=0 .
$$

The equations for $R, S$ can also be written on conservative form, viz.

$$
\begin{aligned}
R_{t}-(c(u) R)_{x} & =-\tilde{c}(u)(R-S)^{2}, \\
S_{t}+(c(u) S)_{x} & =-\tilde{c}(u)(R-S)^{2} .
\end{aligned}
$$

Throughout the paper we will assume that $c$ is a Lipschitz continuous function such that

$$
0<C_{1} \leq c(u) \leq C_{2}, \quad \text { and } \quad 0 \leq c^{\prime}(u) \leq M_{1}
$$

The approach by Zhang and Zheng based on Young measures follows two distinct routes. Either one can use a viscous regularization of the system 1.5 by adding the terms $\epsilon R_{x x}$ and $\epsilon S_{x x}$ to the first and the second equation, respectively, and subsequently analyze in detail the behavior of the solution as $\epsilon \rightarrow 0$, see [12]. Alternatively [11, 13, 14, one can replace the quadratic growth on the right-hand side of equation 1.5 by a linear growth for large values of $R^{2}$ and $S^{2}$. More specifically, introduce the function

$$
Q_{\epsilon}(P)= \begin{cases}\frac{2}{\epsilon}\left(|P|-\frac{1}{2 \epsilon}\right) & \text { for }|P| \geq \frac{1}{\epsilon} \\ P^{2} & \text { for }|P| \leq \frac{1}{\epsilon}\end{cases}
$$

and replace the terms $R^{2}$ and $S^{2}$ by $Q_{\epsilon}(R)$ and $Q_{\epsilon}(S)$, respectively, in the first and the second equation. Again the behavior of the solution has to be analyzed carefully as $\epsilon \rightarrow 0$.

Our approach is based on Young measures for a semi-discrete finite difference upwind scheme. More precisely, introduce a positive discretization parameter $\Delta x$, and approximate $R(j \Delta x, t)$ and $S(j \Delta x, t)$ by $R_{j}(t)$ and $S_{j}(t)$, respectively, that is, $R(j \Delta x, t) \approx R_{j}(t)$ and $S(j \Delta x, t) \approx S_{j}(t), j \in \mathbb{Z}$. Observe that we keep the time variable continuous. The dynamics of $\left(R_{j}(t), S_{j}(t)\right)$ is governed by the system of ordinary differential equations

$$
\begin{aligned}
R_{j}^{\prime}(t)-c_{j+1 / 2}(t) D_{+} R_{j}(t) & =\tilde{c}_{j}(t)\left(R_{j}^{2}(t)-S_{j}^{2}(t)\right), \\
S_{j}^{\prime}(t)+c_{j-1 / 2}(t) D_{-} S_{j}(t) & =-\tilde{c}_{j}(t)\left(R_{j}^{2}(t)-S_{j}^{2}(t)\right),
\end{aligned}
$$


where $D_{ \pm} K_{j}= \pm\left(K_{j \pm 1}-K_{j}\right) / \Delta x$. Furthermore, the functions $c_{j \pm 1 / 2}$ and $\tilde{c}_{j}$ are defined as functions of $R_{j}$ and $S_{j}$, cf. Section 2. The system is augmented by appropriate initial-data. We recover the function $u_{\Delta x}$ by the formula

$$
\int_{0}^{u_{\Delta x}(x, t)} 2 c(u) d u=\int^{x}\left(R_{\Delta x}(\tilde{x}, t)-S_{\Delta x}(\tilde{x}, t)\right) d \tilde{x},
$$

where $R_{\Delta x}$ equals $R_{j}(t)$ on $\left[\left(j-\frac{1}{2}\right) \Delta x,\left(j+\frac{1}{2}\right) \Delta x\right)$, and similarly for $S_{\Delta x}$. We first show that the system possesses solutions that are local in time, and a subsequent a priori estimate turns the local solution into a global one. Once the existence of solutions of the ordinary differential equations has been established, we follow the approach of Zhang and Zheng closely.

Formally, a smooth solution of $(1.8)$ will satisfy the identity

$$
(f(R)+f(S))_{t}-(c(u)(f(R)-f(S)))_{x}=2 \tilde{c} H(R, S),
$$

where

$$
H(R, S)=\frac{1}{2}\left(R^{2}-S^{2}\right)\left(f^{\prime}(R)-f^{\prime}(S)\right)-(f(R)-f(S))(R-S),
$$

for any smooth function $f$. The corresponding discrete relation, Lemma 3.1, is rather more complicated. However, based on this, one shows that the difference scheme keeps the $L^{2}$ norm of $\left\{R_{j}, S_{j}\right\}$ from increasing, cf. Corollary 3.2 a similar result holds in the continuous case as well, cf. [12, Lemma 1]. Intrinsic to the equation is a blow-up property that is not fully understood. Indeed it is known, see [4, that there exist examples with $R$ and $S$ of opposite sign initially, where the solution becomes unbounded. However, if the initial data both are negative initially, one can show that the solution remains regular, see, e.g., [12, Thm. 2]. Henceforth we will make the assumption here that $R$ and $S$ are nonpositive initially. As in the continuous case, [12, Lemma 4], one can show also in the discrete case, Lemma 3.3. that the equation enjoys invariant domains: If $\left(R_{\Delta x}, S_{\Delta x}\right)$ both are nonpositive initially, then they will remain so. Furthermore, if $\left(R_{\Delta x}, S_{\Delta x}\right)$ in addition are bounded from below initially, they will remain so, with the same lower bound. From this it follows that $L^{p}$ norms do not increase, cf. [12, Lemma 5] and Lemma 3.4. Using this one can show in the discrete case, cf. Lemma 3.6, using the ArzelàAscoli theorem, that there exists a function $u$ such that

$$
u_{\Delta x} \rightarrow u \quad \text { uniformly on compacts in } \mathbb{R} \times[0, T] .
$$

The remaining part of the analysis is to show that the limit indeed satisfies the equation. From a priori $L^{p}$ bounds we infer that $R_{\Delta x} \stackrel{\star}{\rightarrow} \bar{R}$ and $S_{\Delta x} \stackrel{\star}{\rightarrow} \bar{S}$ in $L^{\infty}\left([0, T] ; L^{2}(\mathbb{R})\right)$ (recall that $R_{\Delta x}$ equals $R_{j}(t)$ on $\left[\left(j-\frac{1}{2}\right) \Delta x,\left(j+\frac{1}{2}\right) \Delta x\right)$, and similarly for $\left.S_{\Delta x}\right)$, and $\left(R_{\Delta x}-S_{\Delta x}\right)^{2} \rightarrow \overline{\left(R_{\Delta x}-S_{\Delta x}\right)^{2}}$ in $L_{\mathrm{loc}}^{1}\left(\Pi_{T}\right)$. Using the div-curl lemma, Lemma 3.10, and Murat's lemma, Lemma 3.11, we show that $R_{\Delta x} S_{\Delta x} \rightarrow \bar{R} \bar{S}$ in $L_{\text {loc }}^{1}\left(\Pi_{T}\right)$, cf. Lemma 3.15 . Thus we have established that

$$
(\bar{R}-\bar{S})_{t}-(c(u)(\bar{R}+\bar{S}))_{x}=0
$$

holds weakly, cf. 3.51). By direct analysis of the scheme we infer that

$$
c(u)_{x}=2 \tilde{c}(u)(\bar{R}-\bar{S}) \quad \text { weakly, }
$$

cf. (3.49). Using the weak identity $\left(c(u) u_{t}\right)_{x}=\left(c(u) u_{x}\right)_{t}$ we infer that $u_{t}=\frac{1}{2}(\bar{R}+\bar{S})$ holds weakly. To complete the argument, we derive an evolution equation for $\overline{R^{2}}+\overline{S^{2}}$, see Lemma 3.13 (cf. [12, Lemma 11]) to conclude that $u_{t t}-c(u)\left(c(u) u_{x}\right)_{x}=$ 0 weakly, and indeed that $u$ is a weak solution. Our main result can be stated as follows (cf. Theorem 3.19): If $u_{0}$ and $v_{0}$ are such that $R(\cdot, 0)$ and $S(\cdot, 0)$ are nonpositive, and in $L^{3}(\mathbb{R}) \cap L^{1}(\mathbb{R})$, then the semi-discrete difference scheme produces a sequence that converges to a solution of $(1.1)$ in the sense of Definition 1.1 . 
In Appendix A we show a higher integrability result, see Lemma A.1, which reads, here stated in the continuous case (cf. [12, Lemma 5]), as follows:

If $(R(\cdot, 0), S(\cdot, 0)) \in L^{1}(\mathbb{R}) \cap L^{2}(\mathbb{R})$, then $u_{x} \in L_{\text {loc }}^{p}\left(\mathbb{R} \times[0, T], c^{\prime}(u) d x\right)$ for $p \in[2,3)$. The other results in this paper are independent of this, and the significance of the appendix is that it is suspected that such a regularity property could play a role in a uniqueness result.

\section{THE DIFFERENCE SCHEME}

Our first aim is to construct an approximate solution of 1.5 based on a finite difference approximation of the spatial derivative. Rather than work on the full system of three equations, we derive approximate relations for the functions $c(u)$ and $\tilde{c}(u)$ in terms of $R$ and $S$, thereby reducing the system to two equations. The temporal variable will not be discretized, and thus we will consider systems of ordinary differential equations indexed by the spatial lattice and depending on the lattice spacing. Subsequently we will show that as the lattice spacing decreases to zero, the system converges to the solution of 1.5 .

To avoid complicating the convergence analysis, we have chosen to restrict our attention to a semi-discrete difference scheme. To turn the difference scheme into a fully discrete one, we can rely on a variety of different time-discretization techniques, see Section 4 for one example.

We shall use (1.5) as a starting point for a difference scheme. For $j \in \mathbb{Z}$, define $x_{j}=j \Delta x$ and $x_{j+1 / 2}=x_{j}+\frac{1}{2} \Delta x$ where $\Delta x>0$ is the lattice spacing. Let $I_{j}$ denote the interval $\left[x_{j-1 / 2}, x_{j+1 / 2}\right)$.

Given any function $K: \mathbb{R} \rightarrow \mathbb{R}$, we let the value of $K$ at the point $x_{j}$ be denoted by $K_{j}$, that is, $K_{j}=K\left(x_{j}\right)$.

On the other hand, given any sequence $\left\{K_{j}\right\}_{j \in \mathbb{Z}}$, we can consider it as the sampling at lattice points $\Delta x \mathbb{Z}$ of the function $K$ defined by

$$
K(x)=\sum_{j \in \mathbb{Z}} K_{j} \mathbf{1}_{I_{j}}(x) .
$$

Here $\mathbf{1}_{I}$ denotes the characteristic function of the set $I$. Clearly, if values $\left\{K_{j}\right\}$ are computed from some difference scheme, we consider the function (2.1) as the approximation of the true solution.

It is easy to prove the inequalities

$$
\|K\|_{L^{\infty}(\mathbb{R})} \leq \frac{1}{\sqrt{\Delta x}}\|K\|_{L^{2}(\mathbb{R})}, \quad\|K\|_{L^{2}(\mathbb{R})} \leq \frac{1}{\sqrt{\Delta x}}\|K\|_{L^{1}(\mathbb{R})} .
$$

Introduce forward and backward differencing by

$$
D_{ \pm} K_{j}= \pm \frac{1}{\Delta x}\left(K_{j \pm 1}-K_{j}\right)
$$

for any sequence $\left\{K_{j}\right\}$ of real numbers. Let $(R, S)=\left\{\left(R_{j}, S_{j}\right)\right\}_{j \in \mathbb{Z}}$ satisfy the (infinite) system of ordinary differential equations

$$
\begin{aligned}
R_{j}^{\prime}(t)-c_{j+1 / 2}(t) D_{+} R_{j}(t) & =\tilde{c}_{j}(t)\left(R_{j}^{2}(t)-S_{j}^{2}(t)\right), \\
S_{j}^{\prime}(t)+c_{j-1 / 2}(t) D_{-} S_{j}(t) & =-\tilde{c}_{j}(t)\left(R_{j}^{2}(t)-S_{j}^{2}(t)\right),
\end{aligned}
$$

for $j \in \mathbb{Z}$. The functions $c_{j \pm 1 / 2}$ and $\tilde{c}_{j}$ are specified as follows. First set

$$
F(u)=\int_{0}^{u} 2 c(v) d v .
$$


Since $c(u)>0$, we have $F^{\prime}(u)>0$, and $F$ is therefore one-to-one. We start by defining $F_{j-1 / 2}$ by

$$
\left.\begin{array}{c}
\lim _{j \rightarrow-\infty} F_{j-1 / 2}=0, \\
D_{+} F_{j-1 / 2}=R_{j}-S_{j}
\end{array}\right\} \quad \text { or } \quad F_{j+1 / 2}=\Delta x \sum_{i=-\infty}^{j}\left(R_{i}-S_{i}\right) .
$$

Then we can define $u_{j+1 / 2}$ by

$$
u_{j+1 / 2}=\left(F^{-1}\right)\left(F_{j+1 / 2}\right), \quad j \in \mathbb{Z} .
$$

Note that this implies

$$
R_{j}-S_{j}=D_{+} F\left(u_{j-1 / 2}\right)=2 c\left(\bar{u}_{j}^{+}\right) D_{+} u_{j-1 / 2},
$$

for some value $\bar{u}_{j}^{+}$between $u_{j-1 / 2}$ and $u_{j+1 / 2}$. Therefore

$$
D_{+} u_{j-1 / 2}=\frac{R_{j}-S_{j}}{2 c\left(\bar{u}_{j}^{+}\right)} \text {. }
$$

Set

$$
c_{j-1 / 2}=c\left(u_{j-1 / 2}\right),
$$

and note that for some $u_{j}^{+}$between $u_{j-1 / 2}$ and $u_{j+1 / 2}$ we have

$$
D_{+} c_{j-1 / 2}=c^{\prime}\left(u_{j}^{+}\right) D_{+} u_{j-1 / 2}=\frac{c^{\prime}\left(u_{j}^{+}\right)}{2 c\left(\bar{u}_{j}^{+}\right)}\left(R_{j}-S_{j}\right) .
$$

So if we define

$$
\tilde{c}_{j}=\frac{c^{\prime}\left(u_{j}^{+}\right)}{4 c\left(\bar{u}_{j}^{+}\right)}
$$

we have that

$$
D_{+} c_{j-1 / 2}=2 \tilde{c}_{j}\left(R_{j}-S_{j}\right) .
$$

Thus we have defined the functions $c_{j \pm 1 / 2}=c_{j \pm 1 / 2}(R, S)$ and $\tilde{c}_{j}=\tilde{c}_{j}(R, S)$.

We will work with $u_{0} \in H^{1}(\mathbb{R})$ and $v_{0} \in L^{2}(\mathbb{R})$. In this case we define

$$
u_{0, j}=u_{0}(j \Delta x), \quad u_{0, j}^{\prime}=\frac{1}{\Delta x} \int_{I_{j}} u_{0}^{\prime}(x) d x, \quad v_{0, j}=\frac{1}{\Delta x} \int_{I_{j}} v_{0}(x) d x .
$$

The initial values for 2.2 and $(2.3$ are

$$
R_{j}(0)=v_{0, j}+c\left(u_{j, 0}\right) u_{0, j}^{\prime}, \quad \text { and } \quad S_{j}(0)=v_{0, j}-c\left(u_{j, 0}\right) u_{0, j}^{\prime},
$$

for $j \in \mathbb{Z}$. Extend the initial data $\left\{\left(R_{j}(0), S_{j}(0)\right)\right\}_{j \in \mathbb{Z}}$ by, cf. 2.1,

$$
\begin{aligned}
& R_{0, \Delta x}(x)=R_{\Delta x}(x, 0)=\sum_{j} R_{j}(0) \mathbf{1}_{I_{j}}(x), \\
& S_{0, \Delta x}(x)=S_{\Delta x}(x, 0)=\sum_{j} S_{j}(0) \mathbf{1}_{I_{j}}(x) .
\end{aligned}
$$

At this point it is convenient to state the following general lemma.

Lemma 2.1. Let $\varphi$ be a function in $L^{2}(\mathbb{R})$, and set

$$
\varphi_{j}=\frac{1}{\Delta x} \int_{I_{j}} \varphi(x) d x, \quad \varphi_{\Delta x}(x)=\sum_{j} \varphi_{j} \mathbf{1}_{I_{j}}(x) .
$$

Then

as $\Delta x \rightarrow 0$.

$$
\left\|\varphi-\varphi_{\Delta x}\right\|_{L^{2}(\mathbb{R})} \rightarrow 0
$$


Proof. For general functions $\phi, \psi$ in $L^{2}(\mathbb{R})$ we have

$$
\begin{aligned}
\int_{\mathbb{R}}\left(\psi_{\Delta x}(x)-\varphi_{\Delta x}(x)\right)^{2} d x & =\sum_{j} \int_{I_{j}}\left(\frac{1}{\Delta x} \int_{I_{j}}(\psi(z)-\varphi(z)) d z\right)^{2} d x \\
& \leq \sum_{j} \int_{I_{j}} \frac{1}{\Delta x} \int_{I_{j}}(\psi(z)-\varphi(z))^{2} d z d x \\
& =\sum_{j} \int_{I_{j}}(\psi(z)-\varphi(z))^{2} d z \\
& =\int_{\mathbb{R}}(\psi(z)-\varphi(z))^{2} d z .
\end{aligned}
$$

Thus

$$
\begin{aligned}
\left\|\varphi-\varphi_{\Delta x}\right\|_{2} & \leq\|\psi-\varphi\|_{2}+\left\|\psi_{\Delta x}-\varphi_{\Delta x}\right\|_{2}+\left\|\psi-\psi_{\Delta x}\right\|_{2} \\
& \leq 2\|\psi-\varphi\|_{2}+\left\|\psi-\psi_{\Delta x}\right\|_{2}
\end{aligned}
$$

which shows that we, without loss of generality, can assume that $\varphi$ is a smooth function with compact support, $\operatorname{say} \operatorname{supp}(\varphi) \subseteq[-M, M]$. We find, similarly to the derivation of (2.15), that

$$
\begin{aligned}
\int_{\mathbb{R}}\left(\varphi(x)-\varphi_{\Delta x}(x)\right)^{2} d x & =\sum_{j} \int_{I_{j}}\left(\frac{1}{\Delta x} \int_{I_{j}}(\varphi(x)-\varphi(z)) d z\right)^{2} d x \\
& \leq \sum_{j} \int_{I_{j}} \frac{1}{\Delta x} \int_{I_{j}}(\varphi(x)-\varphi(z))^{2} d z d x \\
& \leq 2 \int_{-M-1}^{M+1}\left(\frac{1}{2 \Delta x} \int_{-\Delta x}^{\Delta x}(\varphi(x)-\varphi(x-y))^{2} d y\right) d x
\end{aligned}
$$

Since $\varphi$ is uniformly continuous, we can find $\delta>0$ such that $|y| \leq \delta$ implies

$$
|\varphi(x)-\varphi(x-y)|^{2} \leq \frac{\varepsilon}{4(M+1)}, \quad x \in \mathbb{R} .
$$

By choosing $\Delta x \leq \delta$ we find that

$$
\int_{\mathbb{R}}\left(\varphi(x)-\varphi_{\Delta x}(x)\right)^{2} d x \leq \varepsilon,
$$

which concludes the proof.

This lemma implies

$$
\begin{aligned}
& \left\|R_{0}-R_{0, \Delta x}\right\|_{L^{2}(\mathbb{R})} \rightarrow 0 \quad \text { as } \Delta x \rightarrow 0, \\
& \left\|S_{0}-S_{0, \Delta x}\right\|_{L^{2}(\mathbb{R})} \rightarrow 0 \quad \text { as } \Delta x \rightarrow 0 .
\end{aligned}
$$

Hypothesis 2.2. Consider $u_{0} \in W^{1,3+q}(\mathbb{R}) \cap W^{1,1}(\mathbb{R})$ and $v_{0} \in L^{3+q}(\mathbb{R}) \cap L^{1}(\mathbb{R})$ for some $q>0$, and let $R_{0}$ and $S_{0}$ be defined by 1.6 . Then we assume that $R_{0} \leq 0$ and $S_{0} \leq 0$ almost everywhere.

This assumption implies that also $R_{j}(0)$ and $S_{j}(0)$ are nonpositive for all $j$. Furthermore, by interpolation, we have that $u_{0} \in W^{1, p}(\mathbb{R})$ and $v_{0} \in L^{p}(\mathbb{R})$ for any $p \in[1,3+q]$.

Lemma 2.3. Assume Hypothesis 2.2. Then the system $2.2-(2.3)$ of ordinary differential equations with initial data 2.13 has a unique $C^{1}$ solution $\left\{R_{j}(t)\right\}_{j \in \mathbb{Z}}$ and $\left\{S_{j}(t)\right\}_{j \in \mathbb{Z}}$ for all $t>0$. 
Proof. We use the notation $R(t)=\left\{R_{j}(t)\right\}_{j \in \mathbb{Z}}$ and $S(t)=\left\{S_{j}(t)\right\}_{j \in \mathbb{Z}}$ and write 2.2 and 2.3 , as

$$
\begin{aligned}
R_{j}^{\prime}(t) & =\Psi_{j}^{R}(R, S), \\
S_{j}^{\prime}(t) & =\Psi_{j}^{S}(R, S) .
\end{aligned}
$$

Viewing this as an ordinary differential equation in $\ell^{1}(\mathbb{Z}) \times \ell^{1}(\mathbb{Z})$, where $\ell^{1}(\mathbb{Z})$ denotes the set of absolutely summable sequences with norm

$$
\|v\|_{L^{1}(\mathbb{R})}=\Delta x \sum_{j}\left|v_{j}\right|,
$$

it will have a unique differentiable solution $(R(t), S(t))$ if $\Psi(R, S)=\left\{\left(\Psi_{j}^{R}, \Psi_{j}^{S}\right)\right\}_{j \in \mathbb{Z}}$ is locally Lipschitz continuous. This solution will be defined for $t$ in some interval $\left[0, t^{*}\right)$ where $t^{*}$ is a "blow-up" time, i.e.,

$$
\lim _{t \uparrow t^{*}}\left(\|R(t)\|_{L^{1}(\mathbb{R})}+\|S(t)\|_{L^{1}(\mathbb{R})}\right)=\infty .
$$

If one can show that $\|R(t)\|_{L^{1}(\mathbb{R})}+\|S(t)\|_{L^{1}(\mathbb{R})}<\infty$ for all $t>0$, then a continuously differentiable solution exists for all time.

Now we claim that

$$
\|\Psi(R, S)-\Psi(\hat{R}, \hat{S})\|_{L^{1}(\mathbb{R})} \leq C\left(\|R-\hat{R}\|_{L^{1}(\mathbb{R})}+\|S-\hat{S}\|_{L^{1}(\mathbb{R})}\right),
$$

where $C$ is a constant that depends on $\|(R, S)\|_{L^{1}(\mathbb{R})},\|(\hat{R}, \hat{S})\|_{L^{1}(\mathbb{R})}$ and $\Delta x$. We shall show this for $\Psi^{R}$, the arguments for $\Psi^{S}$ are identical.

To show Lipschitz continuity we start by recalling (cf. 2.5)

$$
F_{j+1 / 2}=F_{j+1 / 2}(R, S)=\Delta x \sum_{i=-\infty}^{j}\left(R_{i}-S_{i}\right) .
$$

Then

$$
\begin{aligned}
& \left|F_{j+1 / 2}(R, S)-F_{j+1 / 2}(\hat{R}, \hat{S})\right| \\
& \quad \leq \Delta x \sum_{i=-\infty}^{j}\left(\left|R_{i}-\hat{R}_{i}\right|+\left|S_{i}-\hat{S}_{i}\right|\right) \leq\|R-\hat{R}\|_{L^{1}(\mathbb{R})}+\|S-\hat{S}\|_{L^{1}(\mathbb{R})},
\end{aligned}
$$

and therefore (writing $\left.\hat{F}_{j}=F_{j+1 / 2}(\hat{R}, \hat{S})\right)$

$$
\|F-\hat{F}\|_{L^{\infty}(\mathbb{R})} \leq\|R-\hat{R}\|_{L^{1}(\mathbb{R})}+\|S-\hat{S}\|_{L^{1}(\mathbb{R})} .
$$

Next we find (cf. 2.2) using 2.6), 2.8), and 2.11) that

$$
\begin{aligned}
\tilde{c}_{j}\left(R_{j}^{2}-S_{j}^{2}\right) & =\frac{1}{2} D_{+} c_{j-1 / 2}\left(R_{j}+S_{j}\right) \\
& =\frac{1}{2}\left(c_{j+1 / 2}-c_{j-1 / 2}\right)\left(R_{j}+S_{j}\right) \\
& =\frac{1}{2}\left(c\left(F_{j+1 / 2}\right)-c\left(F_{j-1 / 2}\right)\right)\left(R_{j}+S_{j}\right),
\end{aligned}
$$

abbreviating $c\left(F_{j \pm 1 / 2}\right)=c\left(\left(F^{-1}\right)\left(F_{j \pm 1 / 2}\right)\right)$. Thus

$$
\begin{aligned}
\tilde{c}_{j}\left(R_{j}^{2}-S_{j}^{2}\right)-\hat{\tilde{c}}_{j}\left(\hat{R}_{j}^{2}-\hat{S}_{j}^{2}\right)= & \frac{1}{2}\left(\left(c\left(F_{j+1 / 2}\right)-c\left(\hat{F}_{j+1 / 2}\right)\right)\left(R_{j}+S_{j}\right)\right. \\
& \quad-\left(c\left(F_{j-1 / 2}\right)-c\left(\hat{F}_{j-1 / 2}\right)\right)\left(R_{j}+S_{j}\right) \\
& \left.+\left(c\left(\hat{F}_{j+1 / 2}\right)-c\left(\hat{F}_{j-1 / 2}\right)\right)\left(R_{j}-\hat{R}_{j}+S_{j}-\hat{S}_{j}\right)\right) .
\end{aligned}
$$


Now

$$
\begin{aligned}
\left|\Psi_{j}^{R}(R, S)-\Psi_{j}^{R}(\hat{R}, \hat{S})\right| \leq \mid c & \left(F_{j+1 / 2}\right)-c\left(\hat{F}_{j+1 / 2}\right)|| D_{+} R_{j} \mid \\
& +c\left(\hat{F}_{j+1 / 2}\right)\left|D_{+}\left(\hat{R}_{j}-R_{j}\right)\right| \\
& +\frac{1}{2}\left|c\left(F_{j+1 / 2}\right)-c\left(\hat{F}_{j+1 / 2}\right)\right|\left(\left|R_{j}\right|+\left|S_{j}\right|\right) \\
& +\frac{1}{2}\left|c\left(F_{j-1 / 2}\right)-c\left(\hat{F}_{j-1 / 2}\right)\right|\left(\left|R_{j}\right|+\left|S_{j}\right|\right) \\
+ & \frac{1}{2}\left|c\left(\hat{F}_{j+1 / 2}\right)-c\left(\hat{F}_{j-1 / 2}\right)\right|\left(\left|R_{j}-\hat{R}_{j}\right|+\left|S_{j}-\hat{S}_{j}\right|\right) \\
\leq & \frac{C}{\Delta x}\left|F_{j+1 / 2}-\hat{F}_{j+1 / 2}\right|\left(\left|R_{j}\right|+\left|R_{j+1}\right|\right) \\
& +\frac{C}{\Delta x}\left(\left|R_{j}-\hat{R}_{j}\right|+\left|R_{j+1}-\hat{R}_{j+1}\right|\right) \\
& +\frac{C}{2 \Delta x}\left|F_{j+1 / 2}-\hat{F}_{j+1 / 2}\right|\left(\left|R_{j}\right|+\left|S_{j}\right|\right) \\
& +\frac{C}{2 \Delta x}\left|F_{j-1 / 2}-\hat{F}_{j-1 / 2}\right|\left(\left|R_{j}\right|+\left|S_{j}\right|\right) \\
& +C\left(\left|R_{j}-\hat{R}_{j}\right|+\left|S_{j}-\hat{S}_{j}\right|\right)
\end{aligned}
$$

since $c$ is Lipschitz continuous functions of $F_{j+1 / 2}$. Multiplying the above by $\Delta x$ and summing over $j$, we see that

$$
\left\|\Psi^{R}(R, S)-\Psi^{R}(\hat{R}, \hat{S})\right\|_{L^{1}(\mathbb{R})} \leq C\left(\|R-\hat{R}\|_{L^{1}(\mathbb{R})}+\|S-\hat{S}\|_{L^{1}(\mathbb{R})}\right)
$$

where we have used 2.22 to find

$$
C=C\left(\Delta x,\|R\|_{L^{1}(\mathbb{R})},\|S\|_{L^{1}(\mathbb{R})},\|\hat{R}\|_{L^{1}(\mathbb{R})},\|\hat{S}\|_{L^{1}(\mathbb{R})}\right) .
$$

Therefore 2.21 holds, and we have established that $\left\{R_{j}(t)\right\}_{j \in \mathbb{Z}}$ and $\left\{S_{j}(t)\right\}_{j \in \mathbb{Z}}$ exist for $t<t^{*}$ (for any initial data). If the initial data are nonpositive and in $L^{1}(\mathbb{R})$, Lemma 3.4 concludes the proof.

Remark 2.4. The existence of global solutions of the system $2.2-2.3$ with initial data 2.12, that is, the fact that $t^{*}=\infty$, follows only after the estimate in Lemma 3.4 , i.e., the inequality (3.13). Thus the results up to Lemma 3.4 are first valid for all times less than $t^{*}$, and only after Lemma 3.4 we can infer that $t^{*}=\infty$. To simplify the notation, we state all these result for all $t$.

For Lemma 2.3 we only require that $u_{0} \in W^{1,1}(\mathbb{R})$ and $v_{0} \in L^{1}(\mathbb{R})$.

\section{Convergence analysis}

Now let $f$ be a sufficiently smooth function, and observe that

$$
f\left(R_{j+1}\right)=f\left(R_{j}\right)+f^{\prime}\left(R_{j}\right)\left(R_{j+1}-R_{j}\right)+\frac{1}{2} f^{\prime \prime}\left(r_{j}\right)\left(R_{j+1}-R_{j}\right)^{2},
$$

where $r_{j}$ is between $R_{j+1}$ and $R_{j}$. This can be rewritten

$$
D_{+} f\left(R_{j}\right)=f^{\prime}\left(R_{j}\right) D_{+} R_{j}+\frac{\Delta x}{2} f^{\prime \prime}\left(r_{j}\right)\left(D_{+} R_{j}\right)^{2} .
$$

Furthermore, we have for any quantity $f_{j}$,

$$
\begin{aligned}
D_{+}\left(c_{j-1 / 2} f_{j}\right) & =c_{j+1 / 2} D_{+} f_{j}+f_{j} D_{+} c_{j-1 / 2} \\
& =c_{j+1 / 2} D_{+} f_{j}+2 f_{j} \tilde{c}_{j}\left(R_{j}-S_{j}\right) .
\end{aligned}
$$


Similarly to 3.1 we have for a sufficiently smooth function $g$

$$
D_{-} g\left(S_{j}\right)=g^{\prime}\left(S_{j}\right) D_{-} S_{j}-\frac{\Delta x}{2} g^{\prime \prime}\left(s_{j}\right)\left(D_{-} S_{j}\right)^{2},
$$

where $s_{j}$ is between $S_{j}$ and $S_{j-1}$. We also have

$$
\begin{aligned}
D_{-}\left(c_{j+1 / 2} g_{j}\right) & =c_{j-1 / 2} D_{-} g_{j}+g_{j} D_{+} c_{j-1 / 2} \\
& =c_{j-1 / 2} D_{-} g_{j}+2 g_{j} \tilde{c}_{j}\left(R_{j}-S_{j}\right) .
\end{aligned}
$$

Next define (cf. 1.11)

$$
H(R, S)=\frac{1}{2}\left(f^{\prime}(R)-f^{\prime}(S)\right)\left(R^{2}-S^{2}\right)-(f(R)-f(S))(R-S) .
$$

We shall use the following lemma repeatedly.

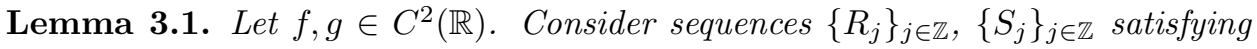
$2.2-2.3$. For $t>0$, there holds

$$
\begin{aligned}
& \frac{d}{d t} f\left(R_{j}\right)-D_{+}\left(c_{j-1 / 2} f\left(R_{j}\right)\right)+\frac{\Delta x}{2} c_{j+1 / 2} f^{\prime \prime}\left(r_{j}\right)\left(D_{+} R_{j}\right)^{2} \\
&=2 \tilde{c}_{j}\left(\frac{1}{2} f^{\prime}\left(R_{j}\right)\left(R_{j}^{2}-S_{j}^{2}\right)-f\left(R_{j}\right)\left(R_{j}-S_{j}\right)\right), \\
& \frac{d}{d t} g\left(S_{j}\right)+D_{-}\left(c_{j+1 / 2} g\left(S_{j}\right)\right)+\frac{\Delta x}{2} c_{j-1 / 2} g^{\prime \prime}\left(s_{j}\right)\left(D_{-} S_{j}\right)^{2} \\
&=-2 \tilde{c}_{j}\left(\frac{1}{2} g^{\prime}\left(S_{j}\right)\left(R_{j}^{2}-S_{j}^{2}\right)-g\left(S_{j}\right)\left(R_{j}-S_{j}\right)\right) .
\end{aligned}
$$

In particular,

$$
\begin{aligned}
\frac{d}{d t}\left(f\left(R_{j}\right)+f\left(S_{j}\right)\right)- & D_{+}\left(c_{j-1 / 2} f\left(R_{j}\right)\right)+D_{-}\left(c_{j+1 / 2} f\left(S_{j}\right)\right) \\
+\frac{\Delta x}{2} & \left(c_{j+1 / 2} f^{\prime \prime}\left(r_{j}\right)\left(D_{+} R_{j}\right)^{2}+c_{j-1 / 2} f^{\prime \prime}\left(s_{j}\right)\left(D_{-} S_{j}\right)^{2}\right) \\
& =2 \tilde{c}_{j} H\left(R_{j}, S_{j}\right),
\end{aligned}
$$

where $r_{j}$ is between $R_{j}$ and $R_{j+1}$, and $s_{j}$ between $S_{j}$ and $S_{j-1}$.

Proof. Multiplying (2.2) by $f_{j}^{\prime}=f^{\prime}\left(R_{j}\right)$, using (3.1) and (3.2), we find that

$$
\begin{aligned}
\frac{d}{d t} f_{j}-D_{+}\left(c_{j-1 / 2} f_{j}\right)+\frac{\Delta x}{2} c_{j+1 / 2} & f^{\prime \prime}\left(r_{j}\right)\left(D_{+} R_{j}\right)^{2} \\
= & 2 \tilde{c}_{j}\left(\frac{1}{2} f_{j}^{\prime}\left(R_{j}^{2}-S_{j}^{2}\right)-f_{j}\left(R_{j}-S_{j}\right)\right),
\end{aligned}
$$

where $f_{j}=f\left(R_{j}\right)$. Similarly, multiplying 2.3 with $g_{j}^{\prime}=g^{\prime}\left(S_{j}\right)$ for some function $g \in C^{2}(\mathbb{R})$, using $(3.3)$ and $(3.4)$, we find that

$$
\begin{aligned}
\frac{d}{d t} g_{j}+D_{-}\left(c_{j+1 / 2} g_{j}\right)+\frac{\Delta x}{2} c_{j-1 / 2} g^{\prime \prime}\left(s_{j}\right)\left(D_{-} S_{j}\right)^{2} & \\
= & -2 \tilde{c}_{j}\left(\frac{1}{2} g_{j}^{\prime}\left(R_{j}^{2}-S_{j}^{2}\right)-g_{j}\left(R_{j}-S_{j}\right)\right),
\end{aligned}
$$

where $g_{j}=g\left(S_{j}\right)$. Choosing $f=g$ and adding (3.8) and (3.9) we conclude that (3.7) holds.

This lemma has several useful consequences, the first of which is the following result. 
Corollary 3.2. We have that

$$
\begin{array}{r}
\Delta x \sum_{j}\left(R_{j}^{2}+S_{j}^{2}\right)(t)+\int_{0}^{T} \Delta x \sum_{j} \Delta x\left(c_{j+1 / 2}\left(D_{+} R_{j}\right)^{2}+c_{j-1 / 2}\left(D_{-} S_{j}\right)^{2}\right) d t \\
\leq \Delta x \sum_{j}\left(R_{j}^{2}+S_{j}^{2}\right)(0) .
\end{array}
$$

In particular, we have

$$
\Delta x \sum_{j}\left(R_{j}^{2}+S_{j}^{2}\right)(t) \leq \Delta x \sum_{j}\left(R_{j}^{2}+S_{j}^{2}\right)(0) .
$$

Proof. Appy Lemma 3.1 with $f(K)=K^{2}$. In this case we observe that $H(R, S)=$ 0 , and $f^{\prime \prime}=2$. Therefore, Lemma 3.1 yields

$$
\begin{aligned}
\frac{d}{d t}\left(R_{j}^{2}+S_{j}^{2}\right) & -D_{+}\left(c_{j-1 / 2} R_{j}^{2}\right)+D_{-}\left(c_{j+1 / 2} S_{j}^{2}\right) \\
& +\Delta x\left(c_{j+1 / 2}\left(D_{+} R_{j}\right)^{2}+c_{j-1 / 2}\left(D_{-} S_{j}\right)^{2}\right) \leq 0 .
\end{aligned}
$$

Multiplying with $\Delta x$, summing over $j$, and integrating in $t$ finishes the proof of the corollary.

The variational wave equation enjoys certain invariance properties in the $(R, S)$ variables. Indeed, if both are nonpositive initially, they will remain so for all time. Furthermore, if in addition the initial data are bounded below by a (negative) constant, then the same constant bounds the solution for all time. See, e.g., 15, Thm. 3.1.6]. The approximate solution has the same properties, which is the result of the following lemma.

Lemma 3.3. The following statements hold:

(i) If $R_{j}(0) \leq 0$ and $S_{j}(0) \leq 0$ for all $j$, then $R_{j}(t) \leq 0$ and $S_{j}(t) \leq 0$ for all $t \geq 0$ and all $j$.

(ii) If $-M \leq R_{j}(0) \leq 0$ and $-M \leq S_{j}(0) \leq 0$ for some positive number $M$ and for all $j$, then $-M \leq R_{j}(t) \leq 0$ and $-M \leq S_{j}(t) \leq 0$ for all $j$ and $t \geq 0$.

Proof. To prove the first statement $(i)$ choose $f(K)=(\max \{0, K\})^{2}$ in (3.7); with this choice

$$
H(R, S)= \begin{cases}0 & \text { if } R S \geq 0 \\ R S(R-S) & \text { if } S<0 \text { and } R>0 \\ R S(S-R) & \text { if } S>0 \text { and } R<0\end{cases}
$$

Hence $H(R, S) \leq 0$, furthermore $f^{\prime \prime}(K) \geq 0$, and thus using (3.7) we find that

$$
\sum_{j}\left(\left(\max \left\{0, R_{j}(t)\right\}\right)^{2}+\left(\max \left\{0, S_{j}(t)\right\}\right)^{2}\right) \leq 0,
$$

since $R_{j}(0) \leq 0$ and $S_{j}(0) \leq 0$ for all $j$. Thus the first statement $(i)$ of the lemma holds.

To prove the second statement $(i i)$ choose $f(K)=(\min \{K+M, 0\})^{2}$. Then we find that

$$
H(R, S)= \begin{cases}0 & \text { if } R \geq-M \text { and } S \geq-M, \\ -2 M(R-S)^{2} & \text { if } R<-M \text { and } S<-M, \\ (R+M)(R-S)(S-M) & \text { if } R<-M \leq S, \\ (S+M)(R-S)(M-R) & \text { if } S<-M \leq R,\end{cases}
$$

which implies that

$$
\left.H(R, S)\right|_{\{(R, S) \mid R<M, S<M\}} \leq 0 .
$$


Furthermore $f^{\prime \prime}(K) \geq 0$. Thus, if $0 \geq R_{j}(0) \geq-M$ and $0 \geq S_{j}(0) \geq-M$, we observe from the first statement $(i)$ that $R_{j}$ and $S_{j}$ remain negative. This implies, using 3.12 , that $H\left(R_{j}, S_{j}\right)(t) \leq 0$. Hence it follows as before, using equation (3.7), that

$$
\sum_{j}\left(\left(\min \left\{0, R_{j}(t)+M\right\}\right)^{2}+\left(\min \left\{0, S_{j}(t)+M\right\}\right)^{2}\right) \leq 0 .
$$

Thus the second statement $(i i)$ of the lemma follows.

In case Hypothesis 2.2 holds, we have the integrability estimate.

Lemma 3.4. If $R_{j}(0) \leq 0$ and $S_{j}(0) \leq 0$ for all $j$, then

$$
\Delta x \sum_{j}\left(\left|R_{j}(t)\right|^{p}+\left|S_{j}(t)\right|^{p}\right) \leq \Delta x \sum_{j}\left(\left|R_{j}(0)\right|^{p}+\left|S_{j}(0)\right|^{p}\right),
$$

for any $p \geq 1$. In addition, if Hypothesis 2.2 holds, then for any $p \in(2,3+q)$

$$
\int_{0}^{T} \Delta x \sum_{j} c^{\prime}\left(u_{j}^{+}\right)\left|D_{+} u_{j-1 / 2}\right|^{p+1} d t \leq C_{p, T},
$$

where $C_{p, T}$ is a constant depending on $p$ and $T$ (but not on $\Delta x$ ).

Remark 3.5. This lemma finishes the proof of Lemma 2.3, namely the fact that $t^{*}=\infty$, cf. Remark 2.4 .

Proof of Lemma 3.4. Choose $f(K)=|K|^{p}$, and observe that

$$
f(0)=0, f(K)=f(-K) \text { and } f^{\prime \prime}(K) \geq 0 .
$$

Now it is easy to see that

$$
H(R, S)=H(S, R) \text { and } H(-S,-R)=-H(R, S) .
$$

Furthermore

We also find that

$$
H(R, R)=H(R,-R)=0
$$

$$
\nabla H(R, S) \cdot(1,1)=\frac{1}{2}\left(f^{\prime \prime}(R)-f^{\prime \prime}(S)\right)\left(R^{2}-S^{2}\right) \geq 0,
$$

since $f^{\prime \prime}$ is an even non-negative function. From this it follows that

$$
\left.H(R, S)\right|_{\{(R, S) \mid R+S \leq 0\}} \leq 0 .
$$

Hence, since $S_{0} \leq 0$ and $R_{0} \leq 0$, by Lemma 3.3 also $R_{j}(t)$ and $S_{j}(t)$ are nonpositive for $t>0$, and thus

$$
\Delta x \sum_{j}\left(f\left(R_{j}(t)\right)+f\left(S_{j}(t)\right)\right) \leq \Delta x \sum_{j}\left(f\left(R_{j}(0)\right)+f\left(S_{j}(0)\right)\right) .
$$

For the proof of $(3.14)$, we fix $p \in(2,3+q)$, remember that $R_{j} \leq 0$ and $S_{j} \leq 0$, and calculate

$$
\begin{aligned}
H\left(R_{j}, S_{j}\right)=\frac{1}{2}\left[p\left(\operatorname{sign}\left(R_{j}\right)\left|R_{j}\right|^{p-1}-\operatorname{sign}\left(S_{j}\right)\left|S_{j}\right|^{p-1}\right)\left(R_{j}^{2}-S_{j}^{2}\right)\right. & \\
& \left.-2\left(\left|R_{j}\right|^{p}-\left|S_{j}\right|^{p}\right)\left(R_{j}-S_{j}\right)\right] \\
=\frac{1}{2} & -p\left(\left|R_{j}\right|^{p-1}-\left|S_{j}\right|^{p-1}\right)\left(\left|R_{j}\right|^{2}-\left|S_{j}\right|^{2}\right) \\
& \left.+2\left(\left|R_{j}\right|-\left|S_{j}\right|\right)\left(\left|R_{j}\right|^{p}-\left|S_{j}\right|^{p}\right)\right] \\
= & \frac{1}{2}\left[-p\left(\left|R_{j}\right|-\left|S_{j}\right|\right)^{2}\left(\left|R_{j}\right|^{p-1}+\left|S_{j}\right|^{p-1}\right)\right.
\end{aligned}
$$




$$
\begin{aligned}
& \underbrace{-2(p-1)\left|R_{j}\right|\left|S_{j}\right|\left(\left|R_{j}\right|-\left|S_{j}\right|\right)\left(\left|R_{j}\right|^{p-2}-\left|S_{j}\right|^{p-2}\right)}_{b\left(R_{j}, S_{j}\right)} \\
& -2\left|R_{j}\right|\left|S_{j}\right|\left(\left|R_{j}\right|-\left|S_{j}\right|\right)\left(\left|R_{j}\right|^{p-2}-\left|S_{j}\right|^{p-2}\right) \\
& \left.+2\left(\left|R_{j}\right|-\left|S_{j}\right|\right)\left(\left|R_{j}\right|^{p}-\left|S_{j}\right|^{p}\right)\right] \\
=\frac{1}{2}\left[-p\left(\left|R_{j}\right|-\left|S_{j}\right|\right)^{2}\left(\left|R_{j}\right|^{p-1}+\left|S_{j}\right|^{p-1}\right)+b\left(R_{j}, S_{j}\right)\right. & \\
& \left.+2\left(\left|R_{j}\right|-\left|S_{j}\right|\right)\left(-\left|R_{j}\right|\left|S_{j}\right|\left(\left|R_{j}\right|^{p-2}-\left|S_{j}\right|^{p-2}\right)+\left|R_{j}\right|^{p}-\left|S_{j}\right|^{p}\right)\right] \\
=\frac{1}{2} & {\left[-p\left(\left|R_{j}\right|-\left|S_{j}\right|\right)^{2}\left(\left|R_{j}\right|^{p-1}+\left|S_{j}\right|^{p-1}\right)+b\left(R_{j}, S_{j}\right)\right.} \\
& \left.+2\left(\left|R_{j}\right|-\left|S_{j}\right|\right)^{2}\left(\left|R_{j}\right|^{p-1}+\left|S_{j}\right|^{p-1}\right)\right] \\
=\frac{1}{2} & {\left[-(p-2)\left(\left|R_{j}\right|-\left|S_{j}\right|\right)^{2}\left(\left|R_{j}\right|^{p-1}+\left|S_{j}\right|^{p-1}\right)+b\left(R_{j}, S_{j}\right)\right] . }
\end{aligned}
$$

It is easy to see that $b(R, S) \leq 0$ for $p>2$, and we also have the inequality

$$
|R|^{p-1}+|S|^{p-1} \geq K_{p}|(|R|-|S|)|^{p-1},
$$

for some positive constant $K_{p}$ depending on $p$. Hence

$$
H\left(R_{j}, S_{j}\right) \leq \frac{-K_{p}(p-2)}{2}\left|R_{j}-S_{j}\right|^{p+1}=-K_{p}(p-2) c\left(\bar{u}_{j}^{+}\right)\left|D_{+} u_{j-1 / 2}\right|^{p+1} .
$$

By Hypothesis 2.2, we find that

$$
\frac{K_{p}(p-2)}{4} \int_{0}^{T} \Delta x \sum_{j} c^{\prime}\left(u_{j}^{+}\right)\left|D_{+} u_{j-1 / 2}\right|^{p+1} \leq \Delta x \sum_{j}\left(\left|R_{j}(0)\right|^{p}+\left|S_{j}(0)\right|^{p}\right) \leq C,
$$

from which (3.14 follows.

Extend the functions $\left(R_{j}, S_{j}\right)$ to the full line, cf. (2.1) and 2.14 , by

$$
R_{\Delta x}(x, t)=\sum_{j} R_{j}(t) \mathbf{1}_{I_{j}}(x), \quad \text { and } \quad S_{\Delta x}(x, t)=\sum_{j} S_{j}(t) \mathbf{1}_{I_{j}}(x) .
$$

Define $F_{\Delta x}$ by

$$
F_{\Delta x}(x, t)=\int^{x}\left(R_{\Delta x}(\tilde{x}, t)-S_{\Delta x}(\tilde{x}, t)\right) d \tilde{x}
$$

and then $u_{\Delta x}$ by

Note that

$$
\int_{0}^{u_{\Delta x}(x, t)} 2 c(u) d u=F_{\Delta x}(x, t)
$$

$$
D_{+} F_{\Delta x}\left(x_{j-1 / 2}, t\right)=R_{j}-S_{j}=D_{+} F\left(u_{j-1 / 2}(t)\right)
$$

or

$$
\int_{u_{j-1 / 2}}^{u_{j+1 / 2}} 2 c(v) d v=\int_{u_{\Delta x}\left(x_{j-1 / 2}, t\right)}^{u_{\Delta x}\left(x_{j+1 / 2}, t\right)} 2 c(v) d v .
$$

Now we have that $\lim _{x \rightarrow-\infty} R_{\Delta x}(x, t)=\lim _{x \rightarrow-\infty} S_{\Delta x}(x, t)=0$, and therefore $\lim _{x \rightarrow-\infty} u_{\Delta x}(x, t)=0$. Hence we must have $u_{\Delta x}\left(x_{j-1 / 2}, t\right)=u_{j-1 / 2}(t)$ for all $j$. It is convenient also to define the piecewise constant function

$$
\bar{u}_{\Delta x}(x, t)=\sum_{j} u_{j-1 / 2}(t) \mathbf{1}_{I_{j-1 / 2}}(x) .
$$

Now we can show the (local) uniform convergence of $u_{\Delta x}$. 
Lemma 3.6. Assume Hypothesis 2.2. Then there exists a function $u \in C\left(\Pi_{T}\right)$ such that for any finite interval $[a, b]$ we have

$$
\lim _{\Delta x \rightarrow 0} u_{\Delta x}(x, t)=u(x, t) \quad \text { uniformly for }(x, t) \in[a, b] \times[0, T] .
$$

Proof. From Hypothesis 2.2 we infer that

$$
\left\|R_{\Delta x}(\cdot, 0)\right\|_{L^{p}(\mathbb{R})}+\left\|S_{\Delta x}(\cdot, 0)\right\|_{L^{p}(\mathbb{R})} \leq C,
$$

for both $p=1$ and $p=2$ for some constant $C$ that is independent of $\Delta x$. From Lemma 3.4 it follows that

$$
\left\|R_{\Delta x}(\cdot, t)\right\|_{L^{1}(\mathbb{R})}+\left\|S_{\Delta x}(\cdot, t)\right\|_{L^{1}(\mathbb{R})} \leq\left\|R_{\Delta x}(\cdot, 0)\right\|_{L^{1}(\mathbb{R})}+\left\|S_{\Delta x}(\cdot, 0)\right\|_{L^{1}(\mathbb{R})} \leq C
$$

where $C$ is the constant in 3.19 . This implies that $F_{\Delta x}$ is uniformly bounded, since

$$
\left|F_{\Delta x}(x, t)\right|=\left|\int^{x}\left(R_{\Delta x}(y, t)-S_{\Delta x}(y, t)\right) d y\right| \leq C .
$$

Next, we observe that

$$
F_{\Delta x}(x, t)-F_{\Delta x}(0, t)=\int_{0}^{x}\left(R_{\Delta x}(y, t)-S_{\Delta x}(y, t)\right) d y .
$$

Therefore, using Cauchy-Schwarz's inequality and (3.20), we find

$$
\begin{aligned}
\left\|F_{\Delta x}(\cdot, t)-F_{\Delta x}(0, t)\right\|_{L^{2}(a, b)}^{2} & =\int_{a}^{b}\left(\int_{0}^{x}\left(R_{\Delta x}(y, t)-S_{\Delta x}(y, t)\right) d y\right)^{2} d x \\
& \leq \int_{a}^{b} 2|x| \int_{\mathbb{R}}\left(R_{\Delta x}^{2}(y, t)+S_{\Delta x}^{2}(y, t)\right) d y d x \\
& \leq C^{2}\left(a^{2}+b^{2}\right) .
\end{aligned}
$$

Similarly, by using 3.16 we find that

$$
\begin{aligned}
\left\|\partial_{x} F_{\Delta x}(\cdot, t)\right\|_{L^{2}(a, b)}^{2} & \leq \int_{a}^{b}\left(R_{\Delta x}(x, t)-S_{\Delta x}(x, t)\right)^{2} d x \\
& \leq 2 \int_{a}^{b}\left(\left|R_{\Delta x}(x, t)\right|^{2}+\left|S_{\Delta x}(x, t)\right|^{2}\right) d x \\
& \leq 2(a+b) C^{2}
\end{aligned}
$$

using (3.11). Thus there is a constant $C_{1}$, independent of $t$ and $\Delta x$ (but depending on $a, b)$, such that

$$
\left\|F_{\Delta x}(\cdot, t)-F_{\Delta x}(0, t)\right\|_{H^{1}(a, b)} \leq C_{1} .
$$

Morrey's inequality now implies that for $x$ and $y$ in $[a, b]$ we have that

$$
\left|F_{\Delta x}(x, t)-F_{\Delta x}(y, t)\right| \leq C_{2}|x-y|^{1 / 2},
$$

for some constant $C_{2}$ which is independent of $\Delta x$ and $t$ (but depending on $a, b$ ).

Now note that using $f(R)=R$ in $(3.8)$ and $g(S)=S$ in $(3.9)$ we find, cf. (1.8), that

$$
\begin{aligned}
R_{j}^{\prime}-D_{+}\left(c_{j-1 / 2} R_{j}\right) & =-\tilde{c}_{j}\left(R_{j}-S_{j}\right)^{2}, \\
S_{j}^{\prime}+D_{-}\left(c_{j+1 / 2} S_{j}\right) & =-\tilde{c}_{j}\left(R_{j}-S_{j}\right)^{2} .
\end{aligned}
$$

Since $D_{+} F_{j}=R_{j}-S_{j}$,

$$
\frac{d}{d t} F_{j}=\Delta x \sum_{i=-\infty}^{j-1}\left(R_{i}^{\prime}-S_{i}^{\prime}\right)=c_{j-1 / 2}\left(R_{j}+S_{j-1}\right) .
$$


Therefore we have that for $0 \leq s \leq t \leq T$

$$
\begin{aligned}
\left\|F_{\Delta x}(\cdot, t)-F_{\Delta x}(\cdot, s)\right\|_{L^{2}(\mathbb{R})} & \leq \int_{s}^{t}\left\|\frac{d}{d t} F_{\Delta x}(\cdot, \tau)\right\|_{L^{2}(\mathbb{R})} d \tau \\
& \leq C_{3} \int_{s}^{t}\left(\left\|S_{\Delta x}(\cdot, \tau)\right\|_{L^{2}(\mathbb{R})}+\left\|R_{\Delta x}(\cdot, \tau)\right\|_{L^{2}(\mathbb{R})}\right) d \tau \\
& \leq C_{3} C|t-s|,
\end{aligned}
$$

using 3.11.

Since $H^{1}(a, b) \subset \subset C(a, b) \subset L^{2}(a, b)$, we can use [9, Lemma 8] to deduce that for $x$ and $y$ in $(a, b)$, we have that for any $\eta>0$, there is a finite $C_{\eta}>0$ such that

$$
\begin{aligned}
\left|F_{\Delta x}(x, t)-F_{\Delta x}(x, s)\right| \leq & \eta\left\|F_{\Delta x}(\cdot, t)-F_{\Delta x}(\cdot, s)\right\|_{H^{1}(a, b)} \\
& \quad+C_{\eta}\left\|F_{\Delta x}(\cdot, t)-F_{\Delta x}(\cdot, s)\right\|_{L^{2}(a, b)} \\
\leq & \eta 2 C_{1}+C_{\eta} C C_{3}|t-s| .
\end{aligned}
$$

For any $\epsilon>0$ we choose $(x, t)$ and $(y, s)$ in $[a, b] \times[0, T]$ and $\eta>0$ such that

$$
C_{2}|x-y|^{1 / 2} \leq \frac{\epsilon}{3}, \quad \eta 2 C_{1} \leq \frac{\epsilon}{3} \quad \text { and then } \quad C_{\eta} C C_{3}|t-s| \leq \frac{\epsilon}{3} .
$$

With this choice

$$
\begin{aligned}
\left|F_{\Delta x}(x, t)-F_{\Delta x}(y, s)\right| & \leq\left|F_{\Delta x}(x, t)-F_{\Delta x}(y, t)\right|+\left|F_{\Delta x}(y, t)-F_{\Delta x}(y, s)\right| \\
& \leq \epsilon .
\end{aligned}
$$

Hence, the sequence $\left\{F_{\Delta x}\right\}_{\Delta x>0}$ is equicontinuous and uniformly bounded in $[a, b] \times$ $[0, T]$, and by the Arzelà-Ascoli theorem there exists a convergent subsequence (which we do not relabel).

By the definition (3.17) of $u_{\Delta x}$ and the assumption on $c$, cf. 1.9, we find that

$$
\left|F_{\Delta x_{j}}(x, t)-F_{\Delta x_{k}}(x, t)\right| \geq C_{4}\left|u_{\Delta x_{j}}(x, t)-u_{\Delta x_{k}}(x, t)\right| \text {, }
$$

for some constant $C_{4}$ depending only on the function $c$. This shows that $\left\{u_{\Delta x_{j}}\right\}$ is Cauchy and thus uniformly convergent on compacts $[a, b] \times[0, T]$.

Remark 3.7. For this lemma to hold it is sufficent to assume that $R_{0}$ and $S_{0}$ (and therefore $\left.R_{\Delta x}(0), S_{\Delta x}(0)\right)$ are nonpositive, and in $L^{1} \cap L^{2}$.

Note that $F_{\Delta x}$ is linear in the interval $I_{j}$ as $R_{\Delta x}$ and $S_{\Delta x}$ are constant there. By definition we have that

$$
\frac{\partial u_{\Delta x}}{\partial F_{\Delta x}}=\frac{1}{2 c\left(u_{\Delta x}\right)}>0 .
$$

This means that $u_{\Delta x}(x, t)$ is monotone in the interval $I_{j}$, and we have that $u_{\Delta x}\left(x_{j \pm 1 / 2}, t\right)=$ $u_{j \pm 1 / 2}(t)$. To simplify the subsequent calculations we introduce

$$
\tilde{u}_{j}=\theta_{j} u_{j-1 / 2}+\left(1-\theta_{j}\right) u_{j+1 / 2}, \quad \theta_{j} \in[0,1], \quad j \in \mathbb{Z},
$$

and

$$
\tilde{u}_{\Delta x}(x, t)=\sum_{j} \tilde{u}_{j}(t) \mathbf{1}_{I_{j}}(x) .
$$

Then for any fixed $x$ and $t$,

$$
\lim _{\Delta x \rightarrow 0} \tilde{u}_{\Delta x}(x, t)=u(x, t) .
$$

This is so since if $x \in I_{j}$, there is a $y_{j} \in I_{j}$ such that $\tilde{u}_{\Delta x}(x, t)=u_{\Delta x}\left(y_{j}, t\right)$ by the monotonicity of $u_{\Delta x}$. Now let $\left\{\Delta x_{k}\right\}$ and $\left\{\Delta x_{\ell}\right\}$ be two sequences tending to zero. Fixing $x$, we can find sequences $\left\{y_{k}\right\}$ and $\left\{y_{\ell}\right\}$ such that $y_{k} \rightarrow x$ and $y_{\ell} \rightarrow x$ and

$$
\tilde{u}_{\Delta x_{k}}(x, t)=u_{\Delta x_{k}}\left(y_{k}, t\right) \quad \text { and } \quad \tilde{u}_{\Delta x_{\ell}}(x, t)=u_{\Delta x_{\ell}}\left(y_{\ell}, t, t\right) \text {. }
$$


Hence

$\left|\tilde{u}_{\Delta x_{k}}(x, t)-\tilde{u}_{\Delta x_{l}}(x, t)\right| \leq\left|u_{\Delta x_{k}}\left(y_{k}, t\right)-u_{\Delta x_{\ell}}\left(y_{k}, t\right)\right|+\left|u_{\Delta x_{\ell}}\left(y_{k}, t\right)-u_{\Delta x_{\ell}}\left(y_{\ell}, t\right)\right|$.

Both terms on the right vanish as $k$ and $\ell$ become large since $u_{\Delta x}$ is uniformly continuous. Hence for any choice of $\left\{\theta_{j}\right\}$, 3.22 holds. In particular, this implies the pointwise convergence

$$
\begin{gathered}
\lim _{\Delta x \rightarrow 0} \sum_{j} u_{j \pm 1 / 2}(t) \mathbf{1}_{I_{j}}(x)=u(x, t) \quad \text { and } \\
\lim _{\Delta x \rightarrow 0} \sum_{j} \tilde{c}_{j}(t) \mathbf{1}_{I_{j}}(x)=\frac{c^{\prime}(u(x, t))}{4 c(u(x, t))}=: \tilde{c}(u(x, t)),
\end{gathered}
$$

uniformly on compacts.

Next, we collect (in three lemmas) some well-known results related to weak convergence. Throughout the paper we use overbars to denote weak limits.

Lemma $3.8([2])$. Let $O$ be a bounded open subset of $\mathbb{R}^{M}$, with $M \geq 1$.

Let $\left\{v_{n}\right\}_{n \geq 1}$ be a sequence of measurable functions on $O$ for which

$$
\sup _{n \geq 1} \int_{O} \Phi\left(\left|v_{n}(y)\right|\right) d y<\infty
$$

for some given continuous function $\Phi:[0, \infty) \rightarrow[0, \infty)$. Then along a subsequence as $n \rightarrow \infty$

$$
g\left(v_{n}\right) \rightarrow \overline{g(v)} \text { in } L^{1}(O)
$$

for all continuous functions $g: \mathbb{R} \rightarrow \mathbb{R}$ satisfying

$$
\lim _{|v| \rightarrow \infty} \frac{|g(v)|}{\Phi(|v|)}=0 .
$$

Let $g: \mathbb{R} \rightarrow(-\infty, \infty]$ be a lower semicontinuous convex function and $\left\{v_{n}\right\}_{n \geq 1}$ a sequence of measurable functions on $O$, for which

$$
v_{n} \rightarrow v \text { in } L^{1}(O), g\left(v_{n}\right) \in L^{1}(O) \text { for each } n, g\left(v_{n}\right) \rightarrow \overline{g(v)} \text { in } L^{1}(O) .
$$

Then

$$
g(v) \leq \overline{g(v)} \text { a.e. on } O .
$$

Moreover, $g(v) \in L^{1}(O)$ and

$$
\int_{O} g(v) d y \leq \liminf _{n \rightarrow \infty} \int_{O} g\left(v_{n}\right) d y
$$

If, in addition, $g$ is strictly convex on an open interval $(a, b) \subset \mathbb{R}$ and

$$
g(v)=\overline{g(v)} \text { a.e. on } O,
$$

then, passing to a subsequence if necessary,

$$
v_{n}(y) \rightarrow v(y) \text { for a.e. } y \in\{y \in O \mid v(y) \in(a, b)\} .
$$

Let $X$ be a Banach space and denote by $X^{\star}$ its dual. The space $X^{\star}$ equipped with the weak- $\star$ topology is denoted by $X_{\text {weak }}^{\star}$, while $X$ equipped with the weak topology is denoted by $X_{\text {weak }}$. According to the Banach-Alaoglu theorem, any bounded ball in $X^{\star}$ is $\sigma\left(X^{\star}, X\right)$-compact. If $X$ separable, then the weak-^ topology is metrizable on bounded sets in $X^{\star}$, and thus one can consider the metric space $C\left([0, T] ; X_{\text {weak }}^{\star}\right)$ of functions $v:[0, T] \rightarrow X^{\star}$ that are continuous with respect to the weak topology. We have $v_{n} \rightarrow v$ in $C\left([0, T] ; X_{\text {weak }}^{\star}\right)$ if $\left\langle v_{n}(t), \phi\right\rangle_{X^{\star}, X} \rightarrow\langle v(t), \phi\rangle_{X^{\star}, X}$ uniformly with respect to $t$, for any $\phi \in X$. The following theorem is a straightforward consequence of the abstract Arzelà-Ascoli theorem: 
Lemma $3.9([2])$. Let $X$ be a separable Banach space, and suppose $v_{n}:[0, T] \rightarrow$ $X^{\star}, n=1,2, \ldots$, is a sequence of measurable functions such that

$$
\left\|v_{n}\right\|_{L^{\infty}\left([0, T] ; X^{\star}\right)} \leq C,
$$

for some constant $C$ independent of $n$. Suppose the sequence

$$
[0, T] \ni t \mapsto\left\langle v_{n}(t), \Phi\right\rangle_{X^{\star}, X}, \quad n=1,2, \ldots,
$$

is equi-continuous for every $\Phi$ that belongs to a dense subset of $X$. Then $v_{n}$ belongs to $C\left([0, T] ; X_{\text {weak }}^{\star}\right)$ for every $n=1,2, \ldots$, and there exists a $v \in C\left([0, T] ; X_{\text {weak }}^{\star}\right)$ such that along a subsequence as $n \rightarrow \infty$

$$
v_{n} \rightarrow v \text { in } C\left([0, T] ; X_{\text {weak }}^{\star}\right) .
$$

Lemma 3.10 (Div-curl lemma [7]). Let $Q \subset \mathbb{R}^{2}$ be a bounded domain. Suppose

$$
\begin{array}{ll}
v_{\varepsilon}^{1} \rightarrow \bar{v}^{1}, & v_{\varepsilon}^{2} \rightarrow \bar{v}^{2}, \\
w_{\varepsilon}^{1} \rightarrow \bar{w}^{1}, & w_{\varepsilon}^{2} \rightarrow \bar{w}^{2},
\end{array}
$$

in $L^{2}(Q)$ as $\varepsilon \rightarrow 0$. Suppose also that the two sequences $\left\{\operatorname{div}\left(v_{\varepsilon}^{1}, v_{\varepsilon}^{2}\right)\right\}_{\varepsilon>0}$ and $\left\{\operatorname{curl}\left(w_{\varepsilon}^{1}, w_{\varepsilon}^{2}\right)\right\}_{\varepsilon>0}$ lie in a common compact subset of $H_{\mathrm{loc}}^{-1}(Q)$, where $\operatorname{div}\left(v_{\varepsilon}^{1}, v_{\varepsilon}^{2}\right)=$ $\partial_{x_{1}} v_{\varepsilon}^{1}+\partial_{x_{2}} v_{\varepsilon}^{2}$ and $\operatorname{curl}\left(w_{\varepsilon}^{1}, w_{\varepsilon}^{2}\right)=\partial_{x_{1}} w_{\varepsilon}^{2}-\partial_{x_{2}} w_{\varepsilon}^{1}$. Then along a subsequence

$$
\left(v_{\varepsilon}^{1}, v_{\varepsilon}^{2}\right) \cdot\left(w_{\varepsilon}^{1}, w_{\varepsilon}^{2}\right) \rightarrow\left(\bar{v}^{1}, \bar{v}^{2}\right) \cdot\left(\bar{w}^{1}, \bar{w}^{2}\right) \text { in } \mathcal{D}^{\prime}(Q) \text { as } \varepsilon \rightarrow 0 .
$$

Lemma 3.11 (Murat's lemma [7]). Suppose that $\left\{\mathcal{L}_{\varepsilon}\right\}_{\varepsilon>0}$ is bounded in $W_{\text {loc }}^{-1, \infty}\left(\Pi_{T}\right)$. Suppose also that $\mathcal{L}_{\varepsilon}=\mathcal{L}_{\varepsilon}^{1}+\mathcal{L}_{\varepsilon}^{2}$, where $\left\{\mathcal{L}_{\varepsilon}^{1}\right\}_{\varepsilon>0}$ lies in a compact subset of $H_{\mathrm{loc}}^{-1}\left(\Pi_{T}\right)$ and $\left\{\mathcal{L}_{\varepsilon}^{2}\right\}_{\varepsilon>0}$ lies in a bounded subset of $\mathcal{M}_{\mathrm{loc}}\left(\Pi_{T}\right)$. Then $\left\{\mathcal{L}_{\varepsilon}\right\}_{\varepsilon>0}$ lies in a compact subset of $H_{\mathrm{loc}}^{-1}\left(\Pi_{T}\right)$.

According to Hypothesis $2.2, R_{0}, S_{0} \in L^{1}(\mathbb{R}) \cap L^{p}(\mathbb{R})$ (with $p>3$ ). In view of 3.10, 3.13 and Lemma 3.8 there exist $R, S \in L^{\infty}\left(0, T ; L^{q}(\mathbb{R})\right), q \in[1, p]$, $\overline{R^{2}}, \overline{S^{2}} \in L^{\infty}\left(0, T ; L^{r}(\mathbb{R})\right), r \in[1, p / 2]$, such that along a subsequence as $\Delta x \rightarrow 0$

$$
\begin{aligned}
& R_{\Delta x} \stackrel{\star}{\rightarrow} R \text { in } L^{\infty}\left(0, T ; L^{2}(\mathbb{R})\right), \quad S_{\Delta x} \stackrel{\star}{\rightarrow} S \text { in } L^{\infty}\left(0, T ; L^{2}(\mathbb{R})\right), \\
& R_{\Delta x}^{2} \rightarrow \overline{R^{2}} \text { in } L^{\infty}\left(0, T ; L^{r}(\mathbb{R})\right), \quad S_{\Delta x}^{2} \stackrel{\star}{S^{2}} \text { in } L^{\infty}\left(0, T ; L^{r}(\mathbb{R})\right) .
\end{aligned}
$$

As a matter of fact, we can assume that for any function $f \in C^{1}(\mathbb{R})$, with

$$
|f(z)| \leq C\left(1+|z|^{2}\right) \text { and }\left|f^{\prime}(z)\right| \leq C(1+|z|)
$$

the following statements hold

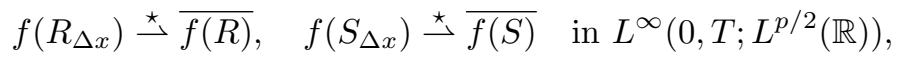

where the same subsequence of $\Delta x \rightarrow 0$ applies to any $f$ from the specified class. Clearly, we can also assume that as $\Delta x \rightarrow 0$

$$
\tilde{c}\left(u_{\Delta x}\right)\left(R_{\Delta x}-S_{\Delta x}\right)^{2} \rightarrow \overline{\tilde{c}(u)(R-S)^{2}}=\tilde{c}(u) \overline{(R-S)^{2}} \quad \text { in } L^{p / 2}\left(\Pi_{T}\right),
$$

by equation (3.23). From Lemmas 3.1 and 3.4 it is not difficult to deduce that the functions

$$
t \mapsto \int_{\mathbb{R}} f\left(R_{\Delta x}\right) \Phi d x, \quad t \mapsto \int_{\mathbb{R}} f\left(S_{\Delta x}\right) \Phi d x
$$

are equi-continuous on $[0, T]$ for every $\Phi \in C_{0}^{\infty}(\mathbb{R})$. In addition, $f\left(R_{\Delta x}\right)$ and $f\left(S_{\Delta x}\right)$ are bounded in $L^{\infty}\left(0, T ; L^{r}(\mathbb{R})\right)$, independently of $\Delta x$. Now we apply Lemma 3.9 with $X^{\star}=L^{r}(\mathbb{R}), X=L^{r^{\prime}}(\mathbb{R})$, and $r^{\prime}=r /(r-1)$. Since $C_{0}^{\infty}(\mathbb{R})$ is dense in $L^{r}(\mathbb{R})$, we can thus assume that $\overline{f(R)}, \overline{f(S)} \in C\left([0, T] ; L_{\text {weak }}^{r}(\mathbb{R})\right)$ and

$$
f\left(R_{\Delta x}\right) \rightarrow \overline{f(R)}, f\left(S_{\Delta x}\right) \rightarrow \overline{f(S)} \text { in } C\left([0, T] ; L_{\text {weak }}^{r}(\mathbb{R})\right) .
$$


Of course, when $f(z)=z$, we can assume $\bar{R}, \bar{S} \in C\left([0, T] ; L_{\text {weak }}^{2}(\mathbb{R})\right)$ and

$$
R_{\Delta x} \rightarrow \bar{R}, S_{\Delta x} \rightarrow \bar{S} \text { in } C\left([0, T] ; L_{\text {weak }}^{2}(\mathbb{R})\right) .
$$

Lemma 3.12. Assume Hypothesis 2.2. Then we have, cf. (1.8),

$$
\bar{R}_{t}-(c(u) \bar{R})_{x}=-\tilde{c}(u) \overline{(R-S)^{2}},
$$

and

$$
\bar{S}_{t}+(c(u) \bar{S})_{x}=-\tilde{c}(u) \overline{(R-S)^{2}},
$$

in the sense of distributions on $\mathbb{R} \times[0, T)$, i.e., for any $\varphi \in C_{0}^{\infty}(\mathbb{R} \times[0, T))$,

$$
\begin{aligned}
\iint_{\Pi_{T}}\left(\bar{R} \varphi_{t}-(c(u) \bar{R}) \varphi_{x}\right) d x d t+\int_{\mathbb{R}} R_{0}(x) \varphi(x, 0) & d x \\
& =\iint_{\Pi_{T}} \tilde{c}(u) \overline{(R-S)^{2}} \varphi d x d t
\end{aligned}
$$

and

$$
\begin{aligned}
\iint_{\Pi_{T}}\left(\bar{S} \varphi_{t}+(c(u) \bar{S}) \varphi_{x}\right) d x d t+\int_{\mathbb{R}} S_{0}(x) \varphi(x, 0) & d x \\
& =\iint_{\Pi_{T}} \tilde{c}(u) \overline{(R-S)^{2}} d x d t .
\end{aligned}
$$

Proof. Fix $\varphi \in C_{0}^{\infty}(\mathbb{R} \times[0, T))$. The equation 3.8 with $f(R)=R$ reads

Set

$$
\frac{d}{d t} R_{j}-D_{+}\left(c_{j-1 / 2} R_{j}\right)=-\tilde{c}_{j}\left(R_{j}-S_{j}\right)^{2} .
$$

$$
\varphi_{j}(t)=\frac{1}{\Delta x} \int_{I_{j}} \varphi(y, t) d y
$$

Next multiply the equation 3.33 with $\varphi_{j}$, sum over $j$, do a partial summation, integrate over $t$, to end up with

$$
\begin{aligned}
-\iint_{\Pi_{T}}\left(R_{\Delta x} \varphi_{t}-c_{\Delta x} R_{\Delta x} \varphi_{x}\right) d x d t & +\int_{\mathbb{R}} R_{0, \Delta x}(x) \varphi(x, 0) d x \\
= & -\iint_{\Pi_{T}} \tilde{c}_{\Delta x}\left(R_{\Delta x}-S_{\Delta x}\right)^{2} \varphi d x d t \\
& +\int_{0}^{T} \sum_{j} c_{j} R_{j} \int_{I_{j}}\left(D_{-} \varphi_{j}-\varphi_{x}\right) d x d t
\end{aligned}
$$

where we have defined the functions $c_{\Delta x}$ and $\tilde{c}_{\Delta x}$ by

$$
c_{\Delta x}(x, t)=\sum_{j} c_{j-1 / 2}(t) \mathbf{1}_{I_{j-1 / 2}}(x) \quad \text { and } \quad \tilde{c}_{\Delta x}(x, t)=\sum_{j} \tilde{c}_{j}(t) \mathbf{1}_{I_{j}}(x) .
$$

By 3.23

Now

$$
c_{\Delta x} \rightarrow c(u), \tilde{c}_{\Delta x} \rightarrow \tilde{c}(u) \text { uniformly on } \operatorname{supp}(\varphi)
$$

$$
\left|D_{-} \varphi_{j}(t)-\varphi_{x}(x, t)\right| \leq\left\|\varphi_{x x}\right\|_{L^{\infty}(\mathbb{R} \times[0, T])} \Delta x, \quad x \in I_{j} .
$$

In view of this and (3.13), the last term in 3.34 is bounded as follows:

$$
\left|\int_{0}^{T} \sum_{j} c_{j} R_{j} \int_{I_{j}}\left(D_{-} \varphi_{j}-\varphi_{x}\right) d x d t\right| \leq C \Delta x \rightarrow 0
$$

Furthermore, in view of 2.20 , as $\Delta x \rightarrow 0$

$$
\int_{\mathbb{R}} R_{0, \Delta x}(x) \varphi(x, 0) d x \rightarrow \int_{\mathbb{R}} R_{0}(x) \varphi(x, 0) d x .
$$


Hence, sending $\Delta x \rightarrow 0$ in 3.34 yields 3.31). The evolution equation 3.32 for $\bar{S}$ is proved in the same way.

We can also prove a generalization of the previous lemma.

Lemma 3.13. Assume Hypothesis 2.2 , and let $f \in C^{2}(\mathbb{R})$ be a convex function satisfying (3.25). Then

$$
\begin{aligned}
& \overline{f(R)}_{t}-(c(u) \overline{f(R)})_{x} \leq 2 \tilde{c}(u)\left(\frac{1}{2} \overline{f^{\prime}(R)\left(R^{2}-S^{2}\right)}-\overline{f(R)(R-S)}\right), \\
& \overline{f(S)}_{t}+(c(u) \overline{f(S)})_{x} \leq-2 \tilde{c}(u)\left(\frac{1}{2} \overline{f^{\prime}(S)\left(R^{2}-S^{2}\right)}-\overline{f(S)(R-S)}\right),
\end{aligned}
$$

in the sense of distributions on $\mathbb{R} \times[0, T)$, i.e., for any $\varphi \in C_{0}^{\infty}(\mathbb{R} \times[0, T)), \varphi \geq 0$,

$$
\begin{gathered}
\iint_{\Pi_{T}}\left(\overline{f(R)} \varphi_{t}-(c(u) \overline{f(R)}) \varphi_{x}\right) d x d t+\int_{\mathbb{R}} f\left(R_{0}(x)\right) \varphi(x, 0) d x \\
\geq-\iint_{\Pi_{T}} 2 \tilde{c}(u)\left(\frac{1}{2} \overline{f^{\prime}(R)\left(R^{2}-S^{2}\right)}-\overline{f(R)(R-S)}\right) \varphi d x d t
\end{gathered}
$$

and

$$
\begin{gathered}
\iint_{\Pi_{T}}\left(\overline{f(S)} \varphi_{t}+(c(u) \overline{f(S)}) \varphi_{x}\right) d x d t+\int_{\mathbb{R}} f\left(S_{0}(x)\right) \varphi(x, 0) d x \\
\geq \iint_{\Pi_{T}} 2 \tilde{c}(u)\left(\frac{1}{2} \overline{f^{\prime}(S)\left(R^{2}-S^{2}\right)}-\overline{f(S)(R-S)}\right) \varphi d x d t .
\end{gathered}
$$

Proof. Similar to the proof of Lemma 3.12, starting from $(3.8$ and 3.9 .

The weak limits $\overline{R^{2}}, \overline{S^{2}}$ satisfy the initial data in a strong sense:

Lemma 3.14. Assume Hypothesis 2.2, Then

$$
\begin{aligned}
& \lim _{t \rightarrow 0+} \int_{\mathbb{R}} \overline{R^{2}} d x=\lim _{t \rightarrow 0+} \int_{\mathbb{R}} \bar{R}^{2} d x=\int_{\mathbb{R}} R_{0}^{2} d x \\
& \lim _{t \rightarrow 0+} \int_{\mathbb{R}} \overline{S^{2}} d x=\lim _{t \rightarrow 0+} \int_{\mathbb{R}} \bar{S}^{2} d x=\int_{\mathbb{R}} S_{0}^{2} d x .
\end{aligned}
$$

Proof. Since $\bar{R}, \bar{S} \in C\left([0, T] ; L_{\text {weak }}^{2}(\mathbb{R})\right)$, it follows from 3.31), 3.32 that

$$
\bar{R}(\cdot, t) \rightarrow R_{0}, \bar{S}(\cdot, t) \rightarrow S_{0} \text { in } L^{2}(\mathbb{R}) \text { as } t \rightarrow 0+.
$$

From this, 3.24, and Lemma 3.8 we conclude that

$$
\int_{\mathbb{R}} R_{0}^{2} d x \leq \liminf _{t \rightarrow 0+} \int_{\mathbb{R}} \bar{R}^{2} d x, \quad \int_{\mathbb{R}} S_{0}^{2} d x \leq \liminf _{t \rightarrow 0+} \int_{\mathbb{R}} \bar{S}^{2} d x .
$$

On the other hand, 3.24 says that $R_{\Delta x}(\cdot, t) \rightarrow \bar{R}(\cdot, t), S_{\Delta x}(\cdot, t) \rightarrow \bar{S}(\cdot, t)$ in $L^{2}(\mathbb{R})$ for a.e. $t>0$, and thereby, using also 3.10 and $(2.20)$,

$$
\int_{\mathbb{R}}\left(\bar{R}^{2}+\bar{S}^{2}\right)(t, x) d x \leq \int_{\mathbb{R}}\left(\overline{R^{2}}+\overline{S^{2}}\right)(t, x) d x \leq \int_{\mathbb{R}}\left(R_{0}^{2}+S_{0}^{2}\right) d x .
$$

Since $\overline{R^{2}}, \overline{S^{2}} \in C\left([0, T] ; L_{\text {weak }}^{r}(\mathbb{R})\right.$ ) (with $r>1$ ), one can prove that this inequality actually holds for all $t>0$. Combining 3.39 and 3.40 yields 3.38 .

Lemma 3.15. Assume Hypothesis 2.2, and let and let $f, g \in C^{2}(\mathbb{R})$ be functions satisfying $|f(z)|,|g(z)| \leq C|z|$. Then

$$
f\left(R_{\Delta x}\right) g\left(S_{\Delta x}\right) \rightarrow \overline{f(R)} \overline{g(S)} \text { in the distributional sense on } \mathbb{R} \times(0, T) .
$$


Proof. We will show that the sequences

$$
\begin{aligned}
& \left\{\partial_{t} f\left(R_{\Delta x}\right)-\partial_{x}\left(c\left(u_{\Delta x}\right) f\left(R_{\Delta x}\right)\right)\right\}_{\Delta x>0}, \\
& \left\{\partial_{t} g\left(S_{\Delta x}\right)+\partial_{x}\left(c\left(u_{\Delta x}\right) g\left(S_{\Delta x}\right)\right)\right\}_{\Delta x>0}
\end{aligned}
$$

are compact in $H_{\mathrm{loc}}^{-1}(\mathbb{R} \times(0, T))$.

Introducing the distribution $\mathcal{L}_{\Delta x}=\partial_{t} f\left(R_{\Delta x}\right)-\partial_{x}\left(c\left(u_{\Delta x}\right) f\left(R_{\Delta x}\right)\right)$, we find

$$
\begin{aligned}
& \left\langle\mathcal{L}_{\Delta x}, \varphi\right\rangle \\
& =-\iint_{\Pi_{T}}\left[2 \tilde{c}_{\Delta x}\left(\frac{1}{2} f^{\prime}\left(R_{\Delta x}\right)\left(R_{\Delta x}^{2}-S_{\Delta x}^{2}\right)-f\left(R_{\Delta x}\right)\left(R_{\Delta x}-S_{\Delta x}\right)+C_{f}\right] \varphi d x d t\right. \\
& \quad+\int_{0}^{T} \sum_{j} c_{j} f\left(R_{j}\right) \int_{I_{j}}\left(D_{-} \varphi_{j}-\varphi_{x}\right) d x d t
\end{aligned}
$$

for $\phi \in C_{0}^{\infty}(\mathbb{R} \times(0, T))$, where $C_{f}(x, t)$ is a function that is bounded in $L^{1}(\mathbb{R} \times(0, T))$ independently of $\Delta x$, cf. (3.34). The last term above is bounded by

$$
p_{\Delta x}:=C\left\|R_{0}\right\|_{L^{2}(\mathbb{R})} \int_{0}^{T}\left\|\sum_{j} D_{-} \varphi_{j} \mathbf{1}_{I_{j}}-\varphi_{x}\right\|_{L^{2}(\mathbb{R})} d t .
$$

Since $\sum_{j} D_{-} \varphi_{j} \mathbf{1}_{I_{j}}$ is a piecewise constant approximation to $\varphi_{x}$, by Lemma 2.1 $p_{\Delta x}$ tends to zero as $\Delta x \rightarrow 0$. Thus we infer that

$$
\left|\left\langle\mathcal{L}_{\Delta x}, \varphi\right\rangle\right| \leq C\|\varphi\|_{L^{\infty}(\mathbb{R} \times(0, T))}+p_{\Delta x}
$$

where $p_{\Delta x}$ tends to zero with $\Delta x$. Thus 3.43 is in a compact subset of $H_{\text {loc }}^{-1}(\mathbb{R} \times$ $(0, T)$ ), while $(3.42)$ is in a bounded subset of the locally bounded Radon measures. Hence Murat's lemma implies that $\mathcal{L}_{\Delta x}$ is compact in $H_{\text {loc }}^{-1}(\mathbb{R} \times(0, T))$. By analogous arguments, $\left\{\partial_{t} g\left(S_{\Delta x}\right)+\partial_{x}\left(c\left(u_{\Delta x}\right) g\left(S_{\Delta x}\right)\right)\right\}$ is compact in $H_{\text {loc }}^{-1}(\mathbb{R} \times(0, T))$.

Now by the div-curl lemma on the sequences

$$
\left\{g\left(S_{\Delta x}\right), c\left(u_{\Delta x}\right) g\left(S_{\Delta x}\right)\right\}_{\Delta x>0} \quad \text { and } \quad\left\{c\left(u_{\Delta x}\right) f\left(R_{\Delta x}\right), f\left(R_{\Delta x}\right)\right\}_{\Delta x>0},
$$

we see that

$$
2 c\left(u_{\Delta x}\right) f\left(R_{\Delta x}\right) g\left(S_{\Delta x}\right) \rightarrow 2 c(u) \overline{f(R)} \overline{g(S)} \quad \text { in the distributional sense, }
$$

which, due to (3.44) and Lemma 3.6, concludes the proof of (3.41).

An immediate consequence of the previous lemma is the following result.

Corollary 3.16. There holds

$$
\overline{(R-S)^{2}}=\overline{R^{2}}-2 \bar{R} \bar{S}+\overline{S^{2}} \quad \text { a.e. in } \mathbb{R} \times(0, T) .
$$

Proof. Since we can assume that $R_{\Delta x} S_{\Delta x} \rightarrow \overline{R S}$ in $L^{1}(\mathbb{R} \times(0, T))$, it follows from Lemma 3.15 that

$$
\iint_{\mathbb{R} \times(0, T)} \overline{R S} \varphi d x d t=\iint_{\mathbb{R} \times(0, T)} \bar{R} \bar{S} \varphi d x d t, \quad \forall \varphi \in C_{0}^{\infty}(\mathbb{R} \times(0, T)),
$$

from which we infer that $\overline{R S}=\bar{R} \bar{S}$ a.e.; Hence 3.45 follows.

Lemma 3.17. Assume Hypothesis 2.2. Then

$$
\overline{R^{2}}=\bar{R}^{2} \quad \text { and } \quad \overline{S^{2}}=\bar{S}^{2}, \quad \text { for a.e. }(x, t) \in \Pi_{T} \text {. }
$$

Consequently, as $\Delta x \rightarrow 0$,

$$
R_{\Delta x} \rightarrow \bar{R}, S_{\Delta x} \rightarrow \bar{S} \text { in } L_{\mathrm{loc}}^{2}\left(\Pi_{T}\right) \text { and almost everywhere in } \Pi_{T} .
$$

Proof. Using Lemmas 3.123 .14 and Corollary 3.45 we can argue exactly as in, e.g., Zhang and Zheng [14, to arrive at 3.46) and (3.47). 
Lemma 3.18. Assume Hypothesis 2.2, Then $u$ is a weak solution of 1.1 , i.e.,

$$
\frac{\partial^{2} u}{\partial t^{2}}-c(u) \frac{\partial}{\partial x}\left(c(u) \frac{\partial u}{\partial x}\right)=0
$$

weakly in $\Pi_{T}$ in the sense that

$$
\iint_{\Pi_{T}}\left(u_{t} \varphi_{t}-c(u)_{x}(c(u) \varphi)_{x}\right) d x d t=0
$$

for all test functions $\varphi \in C_{0}^{\infty}\left(\Pi_{T}\right)$. Here $u_{t}$ and $c(u)_{x}$ are given by (3.53) and (3.49, respectively.

Proof. We claim that

$$
c(u)_{x}=2 \tilde{c}(u)(\bar{R}-\bar{S}), \quad \text { weakly }
$$

To this end let

$$
c_{\Delta x}=\sum_{j} c_{j-1 / 2} \mathbf{1}_{I_{j}},
$$

and compute

$$
\begin{aligned}
\left\langle\frac{\partial}{\partial x} c_{\Delta x}, \varphi\right\rangle & =-\iint_{\Pi_{T}} c_{\Delta x} \varphi_{x} d x d t \\
& =-\int_{0}^{T} \sum_{j} \int_{I_{j}} c_{j-1 / 2} \varphi_{x} d x d t \\
& =-\int_{0}^{T} \sum_{j} c_{j-1 / 2} D_{+} \varphi\left(x_{j-1 / 2}, t\right) \Delta x d t \\
& =\int_{0}^{T} \sum_{j}\left(D_{+} c_{j-1 / 2}\right) \varphi\left(x_{j-1 / 2}, t\right) \Delta x d t \\
& =\int_{0}^{T} \Delta x \sum_{j} \varphi\left(x_{j-1 / 2}, t\right)\left[2 \tilde{c}_{j}\left(R_{j}-S_{j}\right)\right] d t .
\end{aligned}
$$

By sending $\Delta x$ to zero in this equality, and using 3.23 , our claim 3.49 follows.

From Lemma 3.12, we find that

$$
(\bar{R}-\bar{S})_{t}-(c(u)(\bar{R}+\bar{S}))_{x}=0, \quad \text { in the sense of distributions. }
$$

Observe that for a function $u$ that is at least one time differentiable we have that $\left(c(u) u_{t}\right)_{x}=\left(c(u) u_{x}\right)_{t}$ holds in the distributional sense. Specifically, we have

$$
\begin{aligned}
\iint_{\Pi_{T}}\left(c(u) u_{t}\right) \varphi_{x} d x d t & =\iint_{\Pi_{T}} c(u) \varphi_{x} u_{t} d x d t=\iint_{\Pi_{T}}\left((c(u) \varphi)_{x}-c^{\prime}(u) u_{x} \varphi\right) u_{t} d x d t \\
& =\iint\left((c(u) \varphi)_{t}-c^{\prime}(u) u_{t} \varphi\right) u_{x} d x d t \\
& =\iint\left(c(u) u_{x}\right) \varphi_{t} d x d t
\end{aligned}
$$

Thus we see that this can be rewritten

in the sense of distributions. Hence

$$
\frac{\partial}{\partial x}\left(c(u)\left(2 u_{t}-(\bar{R}+\bar{S})\right)\right)=0
$$

$$
u_{t}=\frac{1}{2}(\bar{R}+\bar{S}),
$$

since

$$
\lim _{x \rightarrow-\infty} u(x, t)=\lim _{x \rightarrow-\infty}(\bar{R}(x, t)+\bar{S}(x, t))=0 .
$$


Set

$$
\bar{R}^{\varepsilon}(x, t)=\int_{\mathbb{R}} \bar{R}(y, t) j^{\varepsilon}(x-y) d y,
$$

where $j^{\varepsilon}$ is a standard mollifier. Then

$$
\bar{R}_{t}^{\varepsilon}-\left(c(u) \bar{R}^{\varepsilon}\right)_{x}=-\tilde{c}(u) \overline{(R-S)^{2}} * j^{\varepsilon}+r^{\varepsilon},
$$

where by the DiPerna-Lions folklore lemma

$$
r^{\varepsilon}=(c(u) \bar{R})_{x} * j^{\varepsilon}-\left(c(u) \bar{R}^{\varepsilon}\right)_{x}
$$

tends to zero in $L_{\mathrm{loc}}^{1}\left(\Pi_{T}\right)$. This in turn implies that

$$
\begin{aligned}
\bar{R}_{t}^{\varepsilon}-c(u) \bar{R}_{x}^{\varepsilon} & =-\tilde{c}(u) \overline{(R-S)^{2}} * j^{\varepsilon}+2 \tilde{c}(u)(\bar{R}-\bar{S}) \bar{R}^{\varepsilon}+r^{\varepsilon} \\
& =-\tilde{c}(u)\left(\overline{(R-S)^{2}}-2 \bar{R}^{\varepsilon}(\bar{R}-\bar{S})\right)+r^{\varepsilon} .
\end{aligned}
$$

Similarly, with $\bar{S}^{\varepsilon}=\bar{S} * j^{\varepsilon}$, we get

$$
\bar{S}_{t}^{\varepsilon}+c(u) \bar{S}_{x}^{\varepsilon}=-\tilde{c}(u)\left(\overline{(R-S)^{2}}+2 \bar{S}^{\varepsilon}(\bar{R}-\bar{S})\right)-s^{\varepsilon},
$$

where $s^{\varepsilon}$ tends to zero in $L_{\mathrm{loc}}^{1}\left(\Pi_{T}\right)$. Adding (3.54) and (3.55) and sending $\varepsilon$ to zero, we get, after using Lemma 3.17 ,

$$
\frac{\partial}{\partial t} \frac{1}{2}(\bar{R}+\bar{S})-c(u) \frac{\partial}{\partial x} \frac{1}{2}(\bar{R}-\bar{S})=\tilde{c}(u)\left((\bar{R}-\bar{S})^{2}-\overline{(R-S)^{2}}\right)=0,
$$

which, using 3.53, 3.49) and Lemma 3.15, can be rewritten as 3.48).

We collect some of our results in the following theorem.

Theorem 3.19. Assume $(1.9)$ and Hypothesis 2.2 , Then the semi-discrete difference scheme defined by (2.2)-2.10) produces a sequence that converges to a weak solution of (1.1).

The same conclusion holds if Hypothesis 2.2 is replaced by the assumption that $u_{0} \in W^{1,1}(\mathbb{R})$ and $v_{0} \in L^{1}(\mathbb{R})$ and that $R_{0}$ and $S_{0}$ take values in $[-M, 0]$ for some positive constant $M$.

Proof. Observe that in the case when $R_{0}$ and $S_{0}$ take values in [-M,0], Lemma 3.3 shows that $R_{j}(t), S_{j}(t) \in[-M, 0]$ for all $t$. Thus $R_{\Delta x}, S_{\Delta x} \in L^{\infty} \cap L^{1}$, and by interpolation we see that Hypothesis 2.2 is satisfied.

Remark 3.20. The equation (2.7) is awkward to use in practice, since one must compute the inverse of $F$ at each node. In order to circumvent this we may redefine $\tilde{c}_{j}$ slightly. Let $R_{j}$ and $S_{j}$ be defined as before, but let $u_{j-1 / 2}$ be defined by

$$
D_{+} u_{j-1 / 2}=\frac{R_{j}-S_{j}}{c\left(u_{j-1 / 2}\right)+c\left(u_{j+1 / 2}\right)} .
$$

This is also a nonlinear equation to solve for $u_{j+1 / 2}$, but solving this is likely to be easier than inverting $F$. However, if we accept a certain imbalance, we can define $u_{j+1 / 2}$ by

$$
D_{+} u_{j-1 / 2}=\frac{R_{j}-S_{j}}{2 c\left(u_{j-1 / 2}\right)} .
$$

In order to get our approach to work, it is essential that 2.11 holds. Therefore, we shall define $\tilde{c}_{j}$ so that this is the case. Since $c$ is continuous, we have that

$$
D_{+} c\left(u_{j-1 / 2}\right)=c^{\prime}\left(\bar{u}_{j}\right) D_{+} u_{j-1 / 2},
$$

for some $\bar{u}_{j}$ between $u_{j-1 / 2}$ and $u_{j+1 / 2}$. If $u_{j+1 / 2}$ is defined by (3.56) then we set

$$
\tilde{c}_{j}=\frac{c^{\prime}\left(\bar{u}_{j}\right)}{2\left(c\left(u_{j-1 / 2}\right)+c\left(u_{j+1 / 2}\right)\right)},
$$


while if $u_{j+1 / 2}$ is defined by (3.57) we set

$$
\tilde{c}_{j}=\frac{c^{\prime}\left(\bar{u}_{j}\right)}{4 c\left(u_{j-1 / 2}\right)} .
$$

In both cases 2.11 holds. Therefore, the schemes defined by 2.2), 2.2, and (3.56), 3.58) or (3.57), (3.59) all produce sequences converging to weak solutions of (1.1).

\section{Numerical EXAmples}

The semi-discrete scheme defined by $2.2-2.12$ is rather involved, in particular the computation of the quantity $\tilde{c}_{j}$. For actual computations one needs to make a further discretization of the time variation. We have considered the following versions of the semi-discrete scheme defined by $(2.2)-2.11$. These schemes all use an explicit discretization of (2.2)-2.3),

$$
\begin{aligned}
D_{+}^{t} R_{j}^{n}-c_{j+1 / 2}^{n} D_{+} R_{j}^{n} & =\tilde{c}_{j}^{n}\left(\left(R_{j}^{n}\right)^{2}-\left(S_{j}^{n}\right)^{2}\right), \\
D_{+}^{t} S_{j}^{n}+c_{j-1 / 2}^{n} D_{-} S_{j}^{n} & =-\tilde{c}_{j}^{n}\left(\left(R_{j}^{n}\right)^{2}-\left(S_{j}^{n}\right)^{2}\right),
\end{aligned}
$$

where

$$
c_{j-1 / 2}^{n}=c\left(u_{j-1 / 2}^{n}\right)
$$

and

$$
D_{+}^{t} K_{j}^{n}=\frac{1}{\Delta t}\left(K_{j}^{n+1}-K_{j}^{n}\right) .
$$

Furthermore, since we wish something like 2.11) to hold,

$$
\tilde{c}_{j}^{n}=\frac{D_{+} c_{j-1 / 2}^{n}}{2\left(R_{j}^{n}-S_{j}^{n}\right)} .
$$

The difference between these schemes consists in the way the "cofficients" $c_{j-1 / 2}^{n}$ are defined.

(1) Integration in time. We update $u_{j-1 / 2}^{n}$ by considering a discrete version of 3.53 ,

$$
\begin{aligned}
u_{j+1 / 2}^{n+1}= & u_{j+1 / 2}^{n} \\
& +\frac{\Delta t}{8}\left(R_{j}^{n}+R_{j+1}^{n}+R_{j}^{n+1}+R_{j+1}^{n+1}+S_{j}^{n}+S_{j+1}^{n}+S_{j}^{n+1}+S_{j+1}^{n+1}\right) .
\end{aligned}
$$

We use this scheme since $u$ is discretized on a grid that is staggered with respect to that of $R$ and $S$.

(2) Integration in space. Knowing $u_{-N-1 / 2}^{n}$ for some large $N$, we can set

$$
u_{j+1 / 2}^{n}=u_{j-1 / 2}^{n}+\Delta x \frac{R_{j}^{n}-S_{j}^{n}}{c\left(u_{j-1 / 2}^{n}\right)+c\left(u_{j+1 / 2}^{n}\right)} .
$$

In this section we describe two examples.

Consider first the case where the function $c$ is given by

$$
c(u)=\frac{2}{\pi}(\pi+\arctan (u)),
$$

and the initial data are given by

$$
R(x, 0)=-2 e^{-(x-5)^{2}}, \quad S(x, 0)=2 e^{-(x+5)^{2}} .
$$

In this case

$$
u(x, 0)=\int_{-\infty}^{x} \frac{R(y, 0)-S(y, 0)}{2 c(u(y, 0))} d y, \quad u_{t}(x, 0)=\frac{1}{2}(R(x, 0)+S(x, 0)) .
$$

In Figure 1 we show the computed solution $u$ (top) and $R$ and $S$ (bottom). The 

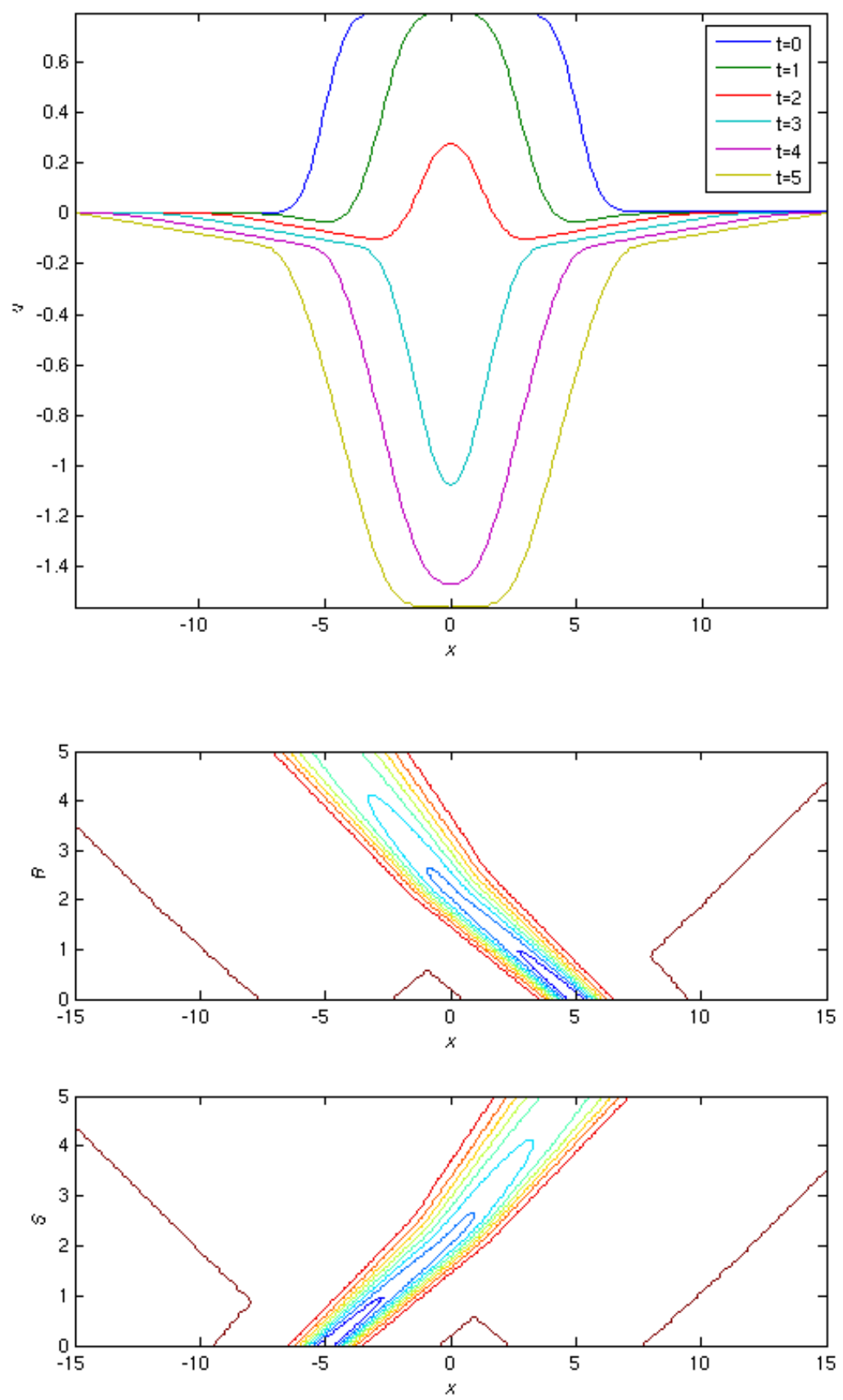

FiguRe 1. The scheme (4.4) with the initial data 4.6 and $c$ given by (4.5). The $u$ variable (top), the $R$ variable (middle) and the $S$ variable (bottom) as functions of $x$ and $t$.

discrete difference scheme can be studied numerically also in cases not covered by the convergence results in this paper. We have included an example of that type 
here. Define the function $c$ by

$$
c(u)=\sqrt{\alpha \cos ^{2}(u)+\beta \sin ^{2}(u)}, \quad \alpha=1.5, \quad \beta=0.5 .
$$

When testing, we take the initial data from [3], and use

$$
u(x, 0)=\frac{\pi}{4}+e^{-x^{2}}, \quad u_{t}(x, 0)=-c(u(x, 0)) \frac{\partial}{\partial x} u(x, 0) .
$$

In order for the two schemes to be compatible, we have defined $u_{j-1 / 2}^{0}$ by

$$
D_{+} u_{j-1 / 2}^{0}=\frac{R_{j}^{0}-S_{j}^{0}}{c\left(u_{j+1 / 2}^{0}\right)+c\left(u_{j-1 / 2}^{0}\right)},
$$

even for the scheme using by (4.3). In Figure 2 we show $u$ for the two methods with initial data (4.8) using $\Delta x=30 / 256$, and $\Delta t=\Delta x$. We remark that using $\Delta t=\Delta x / M$ where $M$ is a large integer, produced very similar results.

\section{Appendix A. Higher integrability properties}

In this appendix we prove a so-called higher integrability result. Briefly stated, we have that if $R_{0}$ and $S_{0}$ are nonpositive and in $L^{1} \cap L^{2}$, then $\partial_{x} u(\cdot, t)$ is in $L_{\text {loc }}^{p}$ for all $p \in[2,3)$. This is obvious if $R_{0}$ and $S_{0}$ are in $L^{3}$, and the significance of this section is that the $\partial_{x} u$ is more integrable than is to be expected. The reason for including this is that we suspect that such a property will play a role in a (yet unknown) uniqueness result.

Throughout the appendix we assume that $R_{0}$ and $S_{0}$ are nonpositive and in $L^{1}(\mathbb{R}) \cap L^{2}(\mathbb{R})$. Then by Lemma 3.4

$$
\Delta x \sum_{j}\left(\left|R_{j}\right|^{1+\alpha}+\left|S_{j}\right|^{1+\alpha}\right) \leq C,
$$

for any $\alpha \in[0,1]$ and some constant $C$ which is independent of $\Delta x$. We also recall that for any smooth function $f$ we have that

$$
\frac{d}{d t} f_{j}-c_{j+1 / 2} D_{+} f_{j}-\frac{\Delta x}{2} f_{j}^{\prime \prime}\left(D_{+} R_{j}\right)^{2}=\tilde{c}_{j} f_{j}^{\prime}\left(R_{j}^{2}-S_{j}^{2}\right)
$$

where $f_{j}=f\left(R_{j}\right), f_{j}^{\prime}=f^{\prime}\left(R_{j}\right)$ and $f_{j}^{\prime \prime}=f^{\prime \prime}\left(r_{j}\right)$ for some $r_{j}$ between $R_{j}$ and $R_{j+1}$.

We now let $\alpha$ be a constant in $[0,1)$ and define $f$ to be a $C^{\infty}$ function such that

$$
\begin{aligned}
f^{\prime}(K) & = \begin{cases}0, & K>-1 / 2, \\
|K|^{\alpha}, & K<-1,\end{cases} \\
f(K) & =\int_{0}^{K} f^{\prime}(\sigma) d \sigma= \begin{cases}0, & K>-1 / 2, \\
\frac{-|K|^{1+\alpha}}{1+\alpha}+C, & K<-1 .\end{cases}
\end{aligned}
$$

Note that $f^{\prime \prime}(K)$ is bounded. Let $\chi(x)$ be a smooth function such that $0 \leq \chi(x) \leq 1$ and

$$
\chi(x)= \begin{cases}0, & x \notin[a-1, b+1], \\ 1, & x \in[a, b],\end{cases}
$$

where $a<b$ are real numbers. Set $\chi_{j}=\chi\left(x_{j}\right)$. We multiply A.1 by $\chi_{j} \Delta x$, sum over $j$ and integrate over $[0, T]$ to end up with

$$
\begin{array}{r}
\left.\Delta x \sum_{j} f_{j} \chi_{j}\right|_{0} ^{T}-\int_{0}^{T} \Delta x \sum_{j} \chi_{j} c_{j+1 / 2} D_{+} f_{j} d t+\int_{0}^{T} \Delta x \sum_{j} \chi_{j} \frac{\Delta x}{2} f_{j}^{\prime \prime}\left(D_{+} R_{j}\right)^{2} d t \\
=\int_{0}^{T} \Delta x \sum_{j} \chi_{j} f_{j}^{\prime} \tilde{c}_{j}\left(R_{j}^{2}-S_{j}^{2}\right) d t .
\end{array}
$$



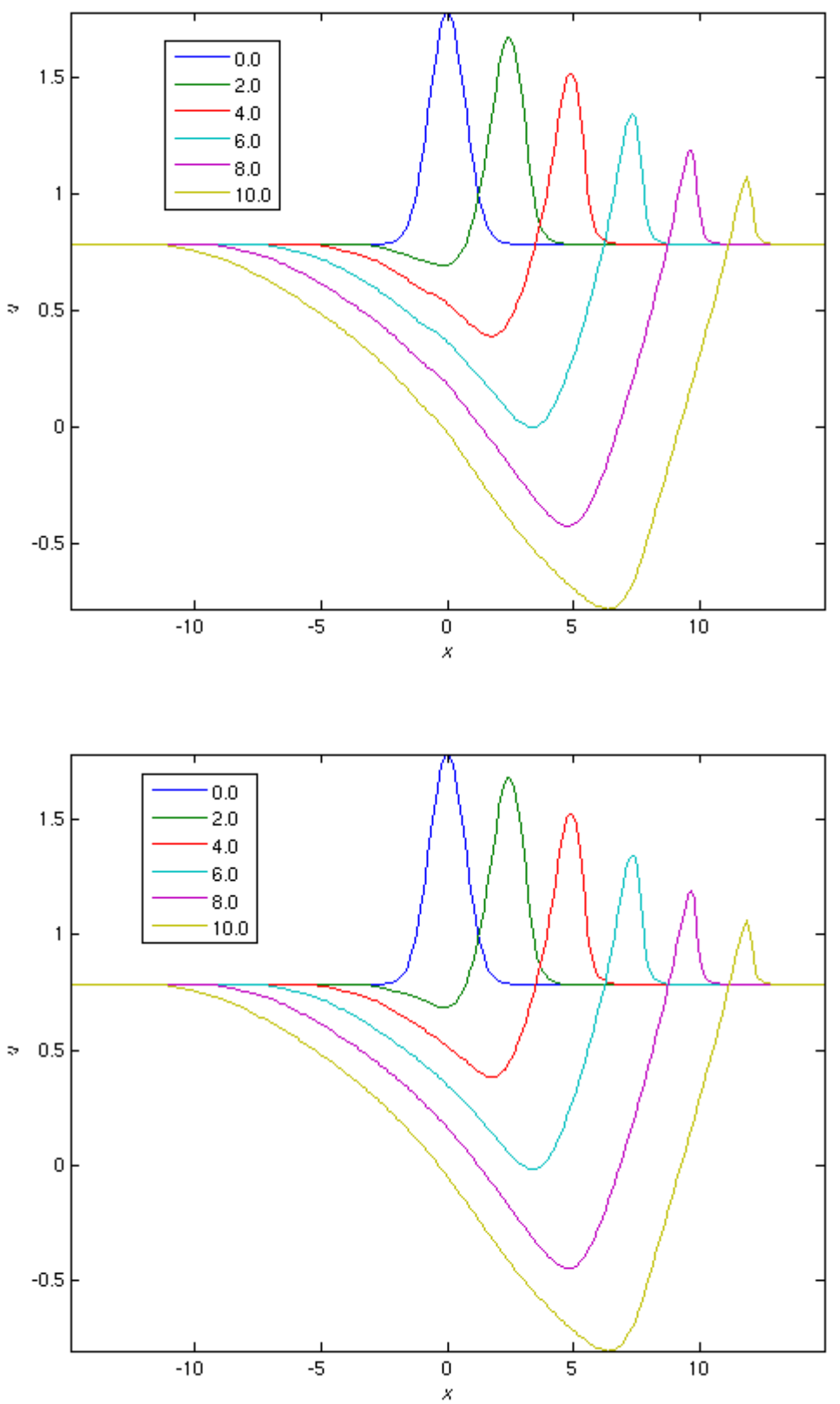

Figure 2. The scheme 4.3) (top) and 4.4) (bottom), with the initial data 4.8) and $c$ given by (4.7).

After a partial summation of the second term on the right, we obtain

$$
\begin{aligned}
\left.\Delta x \sum_{j} f_{j} \chi_{j}\right|_{0} ^{T} & +\int_{0}^{T} \Delta x \sum_{j} f_{j}\left[\chi_{j} 2 \tilde{c}_{j}\left(R_{j}-S_{j}\right)+c_{j-1 / 2} D_{-} \chi_{j}\right] d t \\
& -\int_{0}^{T} \Delta x \sum_{j} \frac{\Delta x}{2} \chi_{j} f_{j}^{\prime \prime}\left(D_{+} R_{j}\right)^{2} d t=\int_{0}^{T} \Delta x \sum_{j} \chi_{j} f_{j}^{\prime} \tilde{c}_{j}\left(R_{j}^{2}-S_{j}^{2}\right) d t .
\end{aligned}
$$


Rearranging this we find that

$$
\begin{aligned}
\int_{0}^{T} \Delta x \sum_{j} \chi_{j} 2 \tilde{c}_{j}[ & \left.\left(R_{j}-S_{j}\right)\left(\frac{-\left|R_{j}\right|^{1+\alpha}}{1+\alpha}\right)-\frac{1}{2}\left(R_{j}^{2}-S_{j}^{2}\right) R_{j}^{\alpha}\right] \mathbf{1}_{\left\{R_{j}<-1\right\}} d t \\
= & -\left.\Delta x \sum_{j} f_{j} \chi_{j}\right|_{0} ^{T}-C \int_{0}^{T} \Delta x \sum_{j} \chi_{j} 2 \tilde{c}_{j}\left(R_{j}-S_{j}\right) d t \\
& \int_{0}^{T} \Delta x \sum_{j} \chi_{j} \tilde{c}_{j}\left[\left(R_{j}^{2}-S_{j}^{2}\right) f_{j}^{\prime}-2\left(R_{j}-S_{j}\right) f_{j}\right] \mathbf{1}_{\left\{R_{j}>-1\right\}} d t \\
& -\int_{0}^{T} \Delta x \sum_{j} \chi_{j} \frac{\Delta x}{2} f_{j}^{\prime \prime}\left(D_{+} R_{j}\right)^{2} d t .
\end{aligned}
$$

By the $L^{p}$ estimates, Lemma 3.4, all terms on the right-hand side of this are bounded by a constant $C_{T, a, b}$ depending only on $T, a, b$ and on the $L^{1}$ and $L^{2}$ norms of $R_{0}$ and $S_{0}$. Furthermore, by the same lemma,

$\left|\int_{0}^{T} \Delta x \sum_{j} \chi_{j} 2 \tilde{c}_{j}\left[\left(R_{j}-S_{j}\right)\left(\frac{-\left|R_{j}\right|^{1+\alpha}}{1+\alpha}\right)-\frac{1}{2}\left(R_{j}^{2}-S_{j}^{2}\right) R_{j}^{\alpha}\right] \mathbf{1}_{\left\{R_{j}>-1\right\}} d t\right| \leq C_{T, a, b}$.

Therefore we get the bound

$$
\left|\int_{0}^{T} \Delta x \sum_{j} \chi_{j} \tilde{c}_{j}\left[\left(R_{j}-S_{j}\right)\left(\frac{-\left|R_{j}\right|^{1+\alpha}}{1+\alpha}\right)-\frac{1}{2}\left(R_{j}^{2}-S_{j}^{2}\right) R_{j}^{\alpha}\right] d t\right| \leq C_{T, a, b},
$$

and similarly

$$
\left|\int_{0}^{T} \Delta x \sum_{j} \chi_{j} \tilde{c}_{j}\left[-\left(R_{j}-S_{j}\right)\left(\frac{-\left|S_{j}\right|^{1+\alpha}}{1+\alpha}\right)-\frac{1}{2}\left(S_{j}^{2}-R_{j}^{2}\right) S_{j}^{\alpha}\right] d t\right| \leq C_{T, a, b} .
$$

Adding these two and recalling that $\left|R_{j}\right|=-R_{j}$ and $\left|S_{j}\right|=-S_{j}$, we get the bound

$$
\begin{aligned}
\mid \int_{0}^{T} \Delta x \sum_{j} \chi_{j} \tilde{c}_{j}\left[\frac{1}{1+\alpha}\right. & \left(\left|R_{j}\right|^{1+\alpha}-\left|S_{j}\right|^{1+a}\right)\left(\left|R_{j}\right|-\left|S_{j}\right|\right) \\
& \left.-\frac{1}{2}\left(\left|R_{j}\right|^{2}-\left|S_{j}\right|^{2}\right)\left(\left|R_{j}\right|^{\alpha}-\left|S_{j}\right|^{\alpha}\right)\right] d t \mid \leq C_{T, a, b} .
\end{aligned}
$$

The term in the square bracket above can be rewritten as

$$
\begin{aligned}
0 \leq \frac{1}{1+\alpha}\left(\left|R_{j}\right|^{1+\alpha}-\left|S_{j}\right|^{1+a}\right)\left(\left|R_{j}\right|-\left|S_{j}\right|\right) & -\frac{1}{2}\left(\left|R_{j}\right|^{2}-\left|S_{j}\right|^{2}\right)\left(\left|R_{j}\right|^{\alpha}-\left|S_{j}\right|^{\alpha}\right) \\
= & \left.\frac{1}{1+\alpha}-\frac{1}{2}\right)\left(\left|R_{j}\right|-\left|S_{j}\right|\right)\left(\left|R_{j}\right|^{1+\alpha}-\left|S_{j}\right|^{1+\alpha}\right) \\
& \quad+\frac{1}{2}\left|R_{j}\right|^{\alpha}\left|S_{j}\right|^{\alpha}\left(\left|R_{j}\right|-\left|S_{j}\right|\right)\left(\left|R_{j}\right|^{1-\alpha}-\left|S_{j}\right|^{1-\alpha}\right) \\
= & \frac{1}{2(1+\alpha)}\left[(1-\alpha)\left(\left|R_{j}\right|-\left|S_{j}\right|\right)\right. \\
& \quad \times\left(\left|R_{j}\right|^{1+\alpha}-\left|S_{j}\right|^{1+\alpha}+\left|R_{j}\right|^{\alpha}\left|S_{j}\right|^{\alpha}\left(\left|R_{j}\right|^{1-\alpha}-\left|S_{j}\right|^{1-\alpha}\right)\right) \\
& \left.\quad+2 \alpha\left(\left|R_{j}\right|-\left|S_{j}\right|\right)\left|R_{j}\right|^{\alpha}\left|S_{j}\right|^{\alpha}\left(\left|R_{j}\right|^{1-\alpha}-\left|S_{j}\right|^{1-\alpha}\right)\right]
\end{aligned}
$$




$$
\begin{aligned}
= & \frac{1-\alpha}{2(1+\alpha)}\left(\left|R_{j}\right|-\left|S_{j}\right|\right)^{2}\left(\left|R_{j}\right|^{\alpha}+\left|S_{j}\right|^{\alpha}\right) \\
& +\frac{\alpha}{1+\alpha}\left(\left|R_{j}\right|-\left|S_{j}\right|\right)\left|R_{j}\right|^{\alpha}\left|S_{j}\right|^{\alpha}\left(\left|R_{j}\right|^{1-\alpha}-\left|S_{j}\right|^{1-\alpha}\right) .
\end{aligned}
$$

Hence, multiplying (A.4) by $2(1+\alpha)$, we arrive at

$$
\begin{aligned}
\int_{0}^{T} \Delta x \sum_{j} \chi_{j} \tilde{c}_{j} & {\left[(1-\alpha)\left(\left|R_{j}\right|-\left|S_{j}\right|\right)^{2}\left(\left|R_{j}\right|^{\alpha}+\left|S_{j}\right|^{\alpha}\right)\right.} \\
& \left.+2 \alpha\left|R_{j}\right|^{\alpha}\left|S_{j}\right|^{\alpha}\left(\left|R_{j}\right|^{1-\alpha}-\left|S_{j}\right|^{1-\alpha}\right)\right] d t \leq C_{T, a, b} .
\end{aligned}
$$

Both terms in the sum and integral above are positive, and thus the integrals of the sums of the individual terms are also bounded.

We can use the inequality

$$
|R|^{\alpha}+|S|^{\alpha} \geq C_{\alpha}|(|R|-|S|)|^{\alpha}=C_{\alpha}|R-S|^{\alpha}
$$

for some constant $C_{\alpha}$ depending on $\alpha$, to get the bound

$$
\int_{0}^{T} \Delta x \sum_{j} \chi_{j} \tilde{c}_{j}\left|R_{j}-S_{j}\right|^{2+\alpha} d t \leq C_{\alpha, T, a, b} .
$$

Since $C_{1}<c(u)<C_{2}$ (cf. $(1.9 p)$ we have proved the following lemma.

Lemma A.1. Let $\alpha \in[0,1)$, and assume that $R_{0}$ and $S_{0}$ are nonpositive, and in $L^{1}(\mathbb{R}) \cap L^{2}(\mathbb{R})$. Then we have the estimate

$$
\int_{0}^{T} \Delta x \sum_{j=j_{a}}^{j_{b}} c^{\prime}\left(u_{j}^{+}\right)\left|D_{+} u_{j-1 / 2}\right|^{2+\alpha} d t \leq C_{\alpha, T, a, b},
$$

where $j_{a} \Delta x \in[a-1, a)$ and $j_{b} \Delta x \in(b, b+1]$.

Acknowledgements. The authors gratefully acknowledge the hospitality of the Mittag-Leffler Institute, Sweden, creating a great working environment for research, during the Fall of 2005.

\section{REFERENCES}

[1] A. Bressan and Y. Zheng. Conservative solutions to a nonlinear variational wave equations. Comm. Math. Phys., 266 (2006) 471-497.

[2] E. Feireisl. Dynamics of Viscous Compressible Fluids. Oxford University Press, Oxford, 2004.

[3] R. T. Glassey, J. K. Hunter, and Y. Zheng. Singularities and oscillations in a nonlinear variational wave equation. In Singularities and Oscillations (J. Rauch, M. Taylor, eds.), Springer, pp. 37-60, 1997.

[4] R. T. Glassey, J. K. Hunter, and Y. Zheng. Singularities of a variational wave equation. J. Differential Equations 129 (1996) 49-78.

[5] H. Holden, K. H. Karlsen, and N. H. Risebro. Convergent difference schemes for the HunterSaxton equation. Math. Comp. 76 (2007) 699-744.

[6] J. K. Hunter and R. A. Saxton. Dynamics of director fields. SIAM J. Appl. Math. 51 (1991) $1498-1521$.

[7] J. Malek, J. Nečas, M. Rokyta and M. Růžička. Weak and measure valued solutions to evolutionary PDEs. Chapman \& Hall, 1996.

[8] R. A. Saxton. Dynamic instability of the liquid crystal director. In Current Progress in Hyperbolic Systems: Riemann Problems and Computations (W. B. Lindquist, ed.), Contemporary Mathematics, vol. 100, American Mathematical Society, Providence, 1989, pp. 325-330.

[9] J. Simon. Compact sets in the space $L^{p}(0, T ; B)$. Ann. Mat. Pura Appl. 146 (1987) 65-96.

[10] P. Zhang and Y. Zheng. On oscillations of an asymptotic equation of a nonlinear variational wave equation. Asymptot. Anal. 18 (1998) 307-327.

[11] P. Zhang and Y. Zheng. Singular and rarefactive solutions to a nonlinear variational wave equation. Chin. Ann. Math. 22B (2001) 159-170. 
[12] P. Zhang and Y. Zheng. Rarefactive solutions to a nonlinear variational wave equation of liquid crystals. Comm. Partial Differential Equations 26 (2001) 381-419.

[13] P. Zhang and Y. Zheng. Weak solutions to a nonlinear variational wave equation. Arch. Rat. Mech. Anal. 166 (2003) 303-319.

[14] P. Zhang and Y. Zheng. Weak solutions to a nonlinear variational wave equation with general data. Ann. Inst. H. Poincaré Anal. Non Linéaire 22 (2005) 207-226.

[15] P. Zhang and Y. Zheng. On the global weak solutions to a variational wave equation. In Handbook of Differential Equations. Evolutionary Equations. Volume 2 (C. M. Dafermos, E. Feireisl, eds.), Elsevier, Amsterdam, 2005, pp. 561-648.

(Holden)

Department of Mathematical Sciences, Norwegian University of Science and Technology, NO-7491 Trondheim, Norway, And

Centre of Mathematics for Applications, University of Oslo, P.O. Box 1053, Blindern, NO-0316 Oslo, Norway

E-mail address: holden@math.ntnu.no

$U R L:$ www.math.ntnu.no/ holden/

(Karlsen)

Centre of Mathematics for Applications, University of Oslo, P.O. Box 1053, Blindern, NO-0316 OSLO, NoRWAY

E-mail address: kennethk@math.uio.no

$U R L:$ www.math.uio.no/ ${ }^{\text {kennethk/ }}$

(Risebro)

Centre of Mathematics for Applications, University of Oslo, P.O. Box 1053, Blindern, NO-0316 OsLO, NORWAY

E-mail address: nilshr@math.uio.no

URL: www . math.uio.no/ nilshr/ 